\title{
BULLET: Particle Swarm Optimization based Scheduling Technique for Provisioned Cloud Resources
}

\author{
Sukhpal Singh Gill ${ }^{1}$, Rajkumar Buyya ${ }^{2}$, Inderveer Chana ${ }^{3}$, Maninder Singh $^{4}$ and Ajith Abraham ${ }^{5}$ \\ ${ }^{1,2}$ CLOUDS Lab, School of Computing and Information Systems, The University of Melbourne, Australia \\ ${ }^{3,4}$ Computer Science and Engineering Department, Thapar University, Patiala, Punjab, India \\ ${ }^{5}$ Machine Intelligence Research Labs (MIR Labs), Scientific Network for Innovation and Research Excellence, Auburn, USA \\ ${ }^{1}$ ssgill@thapar.edu, ${ }^{2}$ rbuyya@unimelb.edu.au , ${ }^{3}$ inderveer@thapar.edu, ${ }^{4}$ msingh@thapar.edu, \\ ajith.abrahamaieee.org
}

\begin{abstract}
Cloud resource scheduling requires mapping of cloud resources to cloud workloads. Scheduling results can be optimized by considering Quality of Service (QoS) parameters as inherent requirements of scheduling. In existing literature, only a few resource scheduling algorithms have considered cost and execution time constraints but efficient scheduling requires better optimization of QoS parameters. The main aim of this research paper is to present an efficient strategy for execution of workloads on cloud resources. A Particle Swarm Optimization (PSO) based resource scheduling technique has been designed named as BULLET which is used to execute workloads effectively on available resources. Performance of the proposed technique has been evaluated in cloud environment. The experimental results show that the proposed technique efficiently reduces execution cost, time and energy consumption along with other QoS parameters.
\end{abstract}

Keywords: Cloud Workload, Cloud Computing, Resource Scheduling, Quality of Service, Particle Swarm Optimization, Energy Consumption, Resource Provisioning

\section{Introduction}

Cloud computing enables resources (Infrastructure, Platform or Software) to be offered as services. These resources are provided using a pay-as-you-use pricing plan [1]. The services offered to the users consist of set of components, which may be offered by different providers. To satisfy the request of customers, service must be provided in accordance with the required level of Quality of Service (QoS). QoS is the capability to guarantee a definite level of performance based on the parameters described by consumer and Service Level Agreement (SLA) in an authorized agreement that describes QoS [2]. One of the major challenges in the current cloud solutions is to provide the required services according to the QoS level expected by the user. Cloud service providers want to confirm that sufficient amount of resources are provisioned to ensure that QoS requirements of cloud service consumers such as deadline, execution time and budget restrictions are met. However, executing too many workloads on a single resource will cause workloads to interfere with each other and result in degraded and unpredictable performance which, in turn, discourages the users [3]. The mapping of workloads to appropriate resources for execution in cloud environment is a complex task and it can be solved by using optimization algorithms. Through these techniques, effective scheduling of resources can be done after resource provisioning. Dispersion, heterogeneity and uncertainty of resources brings challenges to resource allocation, which cannot be satisfied with traditional resource allocation policies in Cloud [4]. Thus, there is a need to make cloud services and cloud-oriented applications efficient by taking care of these properties of the cloud environment. Resource scheduling aims to allocate appropriate resources at the right time to the right workloads, so that applications can utilize the resources effectively which leads to maximization of scaling advantages [17] [18]. The minimum amount of resources should be used for a workload execution to maintain a desirable level of QoS, or minimize workload completion time of a workload. To address this problem, efficient solution should be developed which schedules the provisioned resources efficiently by considering energy consumption, execution cost and execution time as important QoS parameters.

In our earlier work [5] [6] [23], we have identified various research issues related to QoS and SLA for cloud resource scheduling and based on these challenges, we have developed a QoS based resource provisioning technique (Q-aware) to map the resources to the workloads based on user requirements. The main aim of Q-aware is to analyze the workloads, categorize them on the basis of common patterns and then provision the resources for execution of 
cloud workloads before actual resource scheduling. For resource scheduling, resource scheduling framework (QRSF) has been proposed, in which resources have been scheduled by using different resource scheduling policies (cost, time, cost-time and bargaining based). Earlier research work [5] [6] have been implemented in simulated cloud environment and only focus on two QoS parameters (execution cost and time).

The motivation of our research work emerges from the challenge of finding the best resource workload pair according to customer requirements. In real life situations, there are three main QoS constraints that need to be considered for efficient utilization of resources: i) minimizing the execution time and energy consumption, ii) minimizing the execution cost and at the same time meeting the cloud workload deadline and iii) increasing user satisfaction. In this research work, we have extended our previous research work [5] by proposing particle swarm optimization Based resource provisioning and schedULing technique in cLoud EnvironmenT called BULLET which focuses on other QoS parameters (availability, resource utilization, latency and reliability) also along with energy consumption, execution cost and time and requires minimum user involvement during execution of workload. The main objectives of proposed technique are: i) identifying the QoS requirements of a workload, ii) clustering of workloads is done through workload patterns, iii) k-means-based clustering algorithm is used for reclustering of workloads after assigning weights to quality attributes of each workload and iv) resources are provisioned for clustered workloads by the resource provisioner based on their QoS requirements before actual resource scheduling. Further, proposed technique is implemented on real cloud environment using Aneka to validate and optimize QoS parameters.

Initially, resource provisioning takes slight more time to identify the best resources based on QoS requirements of a particular workload, but later on it improves overall efficiency of resource management. Thus, the queuing time and over-utilization and under-utilization of resources can be avoided or be assuaged. Further, proposed technique outperforms as it adjusts the resources at runtime according to the QoS requirements of workload. The paper is structured as follows: In Section 2, related work of resource scheduling along with paper contribution has been presented. PSO based resource scheduling technique has been presented in Section 3. Experimental setup and results has been presented in Section 4. In Section 5, conclusions and the future scope have been presented.

\section{Related Work}

Scheduling of workloads in a cloud environment is challenging due to dynamic and heterogeneous resources spread over geographical area. Most of the reported research deals with workload management systems in a cloud computing environment on the basis of resource requirements. Cloud computing offers dynamic and flexible resource allocation for reliable and guaranteed services in pay according to use fashion. Many cloud consumers can demand number of cloud services concurrently [20] [21]. Subsequently, there is a need to provide all the resources to requesting cloud consumer in a well-organized way to fulfill their requirements.

\subsection{Cloud Resource Scheduling}

Varalakshmi et al. [7] described an OWS (Optimal Workflow based Scheduling) framework to discover a solution that tries to meet the user-desired QoS constraints i.e. execution time. This research shows little improvement in resource utilization and it does not consider cost as one of the QoS parameters. Xing et al. [8] presented an ACO (Ant Colony Optimization) based job scheduling framework, which adapts to dynamic characteristics of Cloud computing and incorporates particular benefits of ACO in NP-hard problems. This approach reduced only job completion time based on pheromone. Topcuoglu et al. [9] presented the HEFT (Heterogeneous Earliest Time First) framework to discover the average execution time of each workload and also the average communication time among the resources of two workloads. The workload with higher rank value is given higher priority. In the resource selection stage workloads are scheduled in priorities and each workload is allocated to the resource that complete the workload at the earliest time. The framework not designed to reduce cost and time. El-kenawy et al. [10] proposed a RASA based scheduling framework to select the jobs based on execution time instead of overall completion time. This technique shows achieving schedules with comparable lower execution time as compared to original Max-Min and RASA (Resource Aware Scheduling Algorithm) by considering only provider's benefit. Lin et al. [11] suggested compromised cost time based resource scheduling policy which considers cost-constrained workflows and taking execution time and cost as QoS parameters. This approach meets user designed deadline and achieve lower cost simultaneously but not considering heterogeneous workflow instances. Verma et al. [12] presented Deadline and Budget Distribution-based Cost-Time Optimization (DBD-CTO) workflow scheduling framework that 
minimizes execution cost while meeting deadline without considering energy consumption and heterogeneous cloud workloads.

\subsection{PSO based Cloud Resource Scheduling}

Pandey et al. [13] introduced a Particle Swarm Optimization (PSO) based heuristic framework (PSO-H) to schedule the applications to Cloud resources that proceeds both computation and data transmission cost. It is used for workflow applications by changing its computation and communication costs. The assessment results show that PSO can reduce the cost and good sharing of workload onto resources. They did not consider execution time of workloads. Selvi et al. [14] presented PSO based resource scheduling mechanism (PSO-HPC) to reduce makespan, price, job rejection ratio and maximize jobs meeting deadline for HPC applications. MATLAB programming environment is used to simulate the HPC applications and resources and verified this technique on Eucalyptus-based cloud environments and results depicted that this technique is efficient in reducing job rejection ratio and execution cost, and improves user's satisfaction without focusing energy consumption. Nuttapong et al. [15] described PSO based scheduling technique (PSO-SW) to achieve scientific workflow execution within the particular deadlines. This approach is used to identify the configuration requirements with minimum cost to execute the particular workflow application and executed the applications with minimum cost without degradation in performance but execution time is not considered as a QoS parameter. Sonia et al. [16] described Dynamic Voltage and Frequency Scaling (DVFS) and PSO based scheduling policy (PSO-DVFS) for scientific workloads to reduce consumption of power in which different levels of voltage supply workloads are used through sacrificing clock frequencies. This multiple voltage involves a compromise between the quality of schedules and energy but execution time and cost are not considered as a QoS parameter. Proposed technique (BULLET) has been compared with existing resource scheduling techniques as described in Table 1.

Table 1: Comparison of Proposed Technique With Existing Resource Scheduling Techniques

\begin{tabular}{|c|c|c|c|c|}
\hline Technique & $\begin{array}{l}\text { Provisioning based } \\
\text { Scheduling }\end{array}$ & Workload Type & Clustering of Workloads & QoS Parameters \\
\hline OWS [7] & $x$ & Homogenous & $x$ & Execution Time \\
\hline ACO [8] & $x$ & Homogenous & $x$ & Completion Time \\
\hline HEFT [9] & $x$ & Homogenous & $x$ & Communication Time \\
\hline RSA [10] & $x$ & $\begin{array}{l}\text { Homogenous and } \\
\text { Heterogeneous }\end{array}$ & $x$ & Execution Time \\
\hline CTC [11] & $x$ & Homogenous & $x$ & Communication Cost \\
\hline DBD-CTO [12] & $x$ & Homogenous & $x$ & Execution Time \\
\hline PSO-H [13] & $x$ & Homogenous & $x$ & Computation and communication cost \\
\hline PSO-HPC [14] & $x$ & $\begin{array}{l}\text { Homogenous and } \\
\text { Heterogeneous }\end{array}$ & $x$ & Execution cost \\
\hline PSO-SW [15] & $x$ & Homogenous & $x$ & Execution Time \\
\hline PSO-DVFS [16] & $x$ & Homogenous & $x$ & Energy \\
\hline $\begin{array}{l}\text { BULLET } \\
\text { (Proposed) }\end{array}$ & $\sqrt{ }$ & $\begin{array}{l}\text { Homogenous and } \\
\text { Heterogeneous }\end{array}$ & $\sqrt{ }$ & $\begin{array}{l}\text { Execution Time, cost, energy, availability, } \\
\text { resource utilization, latency and reliability }\end{array}$ \\
\hline
\end{tabular}

These research works have considered only one of the QoS parameters from energy, cost and time but all the three parameters have not been considered simultaneously in any of the existing work to the best of the knowledge of the authors. Moreover, most of the existing work considers homogeneous cloud workloads. PSO based resource scheduling technique considers the basic features of cloud computing in order to execute the heterogeneous cloud workloads with minimum execution cost, time and energy consumption along with other QoS parameters.

\subsection{Our Contributions}

We present a PSO based resource scheduling technique for both homogenous and heterogeneous cloud workloads. This is an extension of our previous work [5]. The proposed technique focuses on how to map the cloud workload in order to improve execution cost, time and energy along with other QoS parameters (availability, resource utilization, latency and reliability). The proposed technique has been evaluated in real cloud environment. Aneka application development platform is used as a scalable cloud middleware to make interaction between SaaS and IaaS to deploy proposed technique on real cloud environment. The performance of proposed technique has also been tested on cloud testbed using synthetic workloads for different QoS parameters. We have then compared the experimental results of proposed technique with existing PSO based resource scheduling techniques. The main contribution of this paper is: i) scheduling technique for effective management of resources is proposed, ii) used real cloud platform to deploy proposed technique, iii) performance of proposed technique has been evaluated in cloud environment iv) 
optimized important QoS parameters such as execution cost, energy consumption and execution time and v) improved the customer satisfaction and queuing time, over and under-utilization of resources can be avoided or be assuaged.

\section{BULLET: Proposed PSO Based Resource Scheduling Technique}

In cloud computing, resource scheduling is the core of resource management system. It essentially indicates mapping of cloud workloads to the appropriate resources from the available resource pool. This process searches the best resource and maps with cloud workload based on consumer requirements. Process of resource scheduling comprises of four steps. In First step, workloads are analyzed and clustered based on their requirements. Second step, identifies the required set of resources from resource pool. Third step, maps the cloud workload with appropriate resources based on QoS requirements as specified by user. In Final step, schedule the resources to execute workloads therefore further guaranteeing near optimal satisfaction of QoS requirements. Need of optimized resource scheduling in cloud is achieved using proposed technique. For example, assume that a customer wants to purchase some items from grocery store, then salesman would ask the requirements in terms of budget etc. and then salesman will display the items accordingly. Based on the money they want to spend and other requirements and constraints, select the particular item among all the displayed items. Figure 1 shows the architecture of proposed technique.

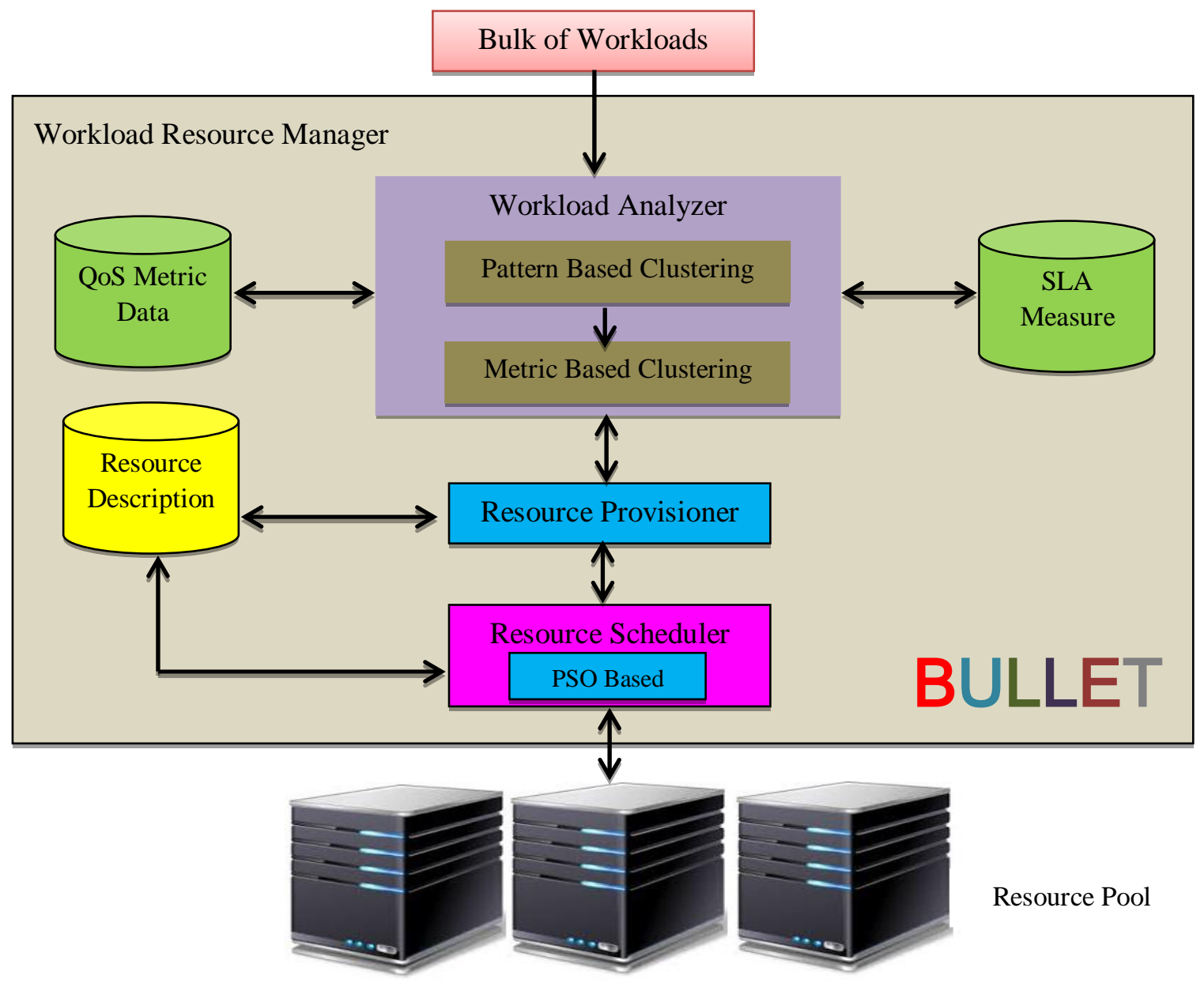

Figure 1: Architecture of BULLET

\subsection{Resource Provisioning}

The resource provisioning technique comprises of following units: 
i) Bulk of Workloads: Bulk of Workloads (BoW) are coming for execution and are processed and stored in workload queue.

ii) Workload Resource Manager: Workload Resource Manager (WRM) contains the information about resources, QoS metrics and SLA to provision the resources for execution of workloads based on QoS requirements described by cloud consumer.

iii) SLA Measure: WRM receipts the information from the suitable Service Level Agreement (SLA). After studying and confirming the various QoS constraints which the workload has required, WRM checking the availability of resources.

iv) QoS Metric Data: It contains the information regarding QoS metrics used to calculate weight for clustering of workloads.

v) Workload Analyzer: The aim of Workload Analyzer is to look at different characteristics of a cloud workload to determine the feasibility of porting the application in the cloud. The different cloud workloads have different set of QoS requirements and characteristics. All the workloads are submitted to WRM are analyzed based on their QoS requirements. For QoS, the required workload patterns are identified for clustering of workloads then identifies the metrics required to assign the weights based on level of measurement (Section 3.1.1) described in QoS requirements specified in SLA. K-Means based clustering algorithm is used for re-clustering the workloads for execution on different set of resources.

vi) Resource Information: The resource details include the number of CPU using, size of memory, cost of resources, type of resources and number of resources. All the common resources are stored in resource pool.

vii) Resource Provisioner: It provides the demanded resources to the workload for their execution in cloud environment only if required resources are available in resource pool. If the required resources are not available according to QoS requirement then the WRM asks to resubmit the workload with QoS requirement in the form of SLA. After the provisioning of resources, workloads are submitted to resource scheduler. Then the resource scheduler will ask to submit the workload for resources provisioned. After this resource scheduler send back the results to WRM, cloud workload contains the resource information.

viii) Resource Scheduler: It will execute all the workloads on provisioned resources efficiently and described in Section 3.2.

Cloud workload is an abstraction of work of that instance or set of instances executing on the appropriate resources with different QoS requirements submitted by cloud consumer as a type of application. The types of workload that have been considered for this research work are: websites, technological computing, endeavor software, performance testing, online transaction processing, e-commerce, central financial services, storage and backup services, production applications, software/project development and testing, graphics oriented, critical internet applications and mobile computing services [5] [6].

\subsubsection{Clustering of Workloads}

Based on the important features of Cloud workloads and workload patterns the clustering of Cloud workloads has been done and process of clustering has been described in our previous research work in detail [5]. The outcome of pattern based workload clustering is shown in Table 2 .

Table 2: Cloud Workloads and Their QoS Requirements After Pattern Based Clustering

\begin{tabular}{|l|l|}
\hline \multicolumn{1}{|c|}{ Workload } & \multicolumn{1}{c|}{ QoS Requirements } \\
\hline Websites & Reliable storage, High network bandwidth, High availability \\
\hline Technological Computing & Computing capacity, Reliable storage \\
\hline Endeavour Software & Security, High availability, Customer Confidence Level, Correctness \\
\hline Performance Testing & Execution time, energy consumption and execution cost \\
\hline Online Transaction Processing & Security, High availability, Internet accessibility, Usability \\
\hline E-Com & Variable computing load, Customizability \\
\hline Central Financial Services & Security, High availability, Changeability, Integrity \\
\hline Storage and Backup Services & Reliability, Persistence \\
\hline Productivity Applications & Network bandwidth, Latency, Data backup, Security \\
\hline $\begin{array}{l}\text { Software/Project Development and } \\
\text { Testing }\end{array}$ & User self-service rate, Flexibility, Creative group of infrastructure services, Testing time \\
\hline Graphics Oriented & Network bandwidth, Latency, Data backup, Visibility \\
\hline Critical Internet Applications & High availability, Serviceability, Usability \\
\hline Mobile Computing Services & High availability, Reliability, Portability \\
\hline
\end{tabular}


Further, $K$-means based clustering algorithm is used for re-clustering the workloads for execution on different set of resources and process of clustering using $K$-means based clustering algorithm has been described in our previous research work in detail [6]. Final set of workloads is shown in Table 3.

Table 3: K-Means Based Clustering of Workloads

\begin{tabular}{|c|c|c|}
\hline Cluster & Cluster Name & Workloads \\
\hline C1 & Compute & Technological Computing, Performance Testing \\
\hline C2 & Storage & E-Com and Storage and Backup Services \\
\hline C3 & Communication & Websites, Critical Internet Applications, Mobile Computing Services \\
\hline C4 & Administration & $\begin{array}{c}\text { Endeavour Software, Online Transaction Processing, Central Financial Services, Productivity } \\
\text { Applications, Software/Project Development and Testing and Graphics Oriented }\end{array}$ \\
\hline
\end{tabular}

\subsection{Resource Scheduling}

We have designed Particle Swarm Optimization (PSO) based resource scheduling algorithm by considering different QoS parameters (Execution Time, Cost and Energy Consumption).

\subsubsection{Requirements}

Following are some important requirements to design an efficient resource scheduling algorithm:

Efficiency: Provisioning of resources offers the facility to reduce the cloud overheads which requires QoS based efficient management of resources.

Efficient Resource Usage: Efficient scheduling of resources should minimize wastage of the resources. Different cloud workloads are waiting for execution should be executed with maximum resource utilization of resources and optimizing QoS parameters (execution time and cost).

Fair Scheduling: The number of resources allotted to every consumer should be independent of number of cloud workloads each user submits.

Adaptability and scalability: A smart scheduler adapts as per the resources, i.e., whenever resources join or leave (dynamically), it manages the resources and workloads' execution process efficiently.

\subsubsection{Problem formulation}

Cloud resource scheduling is a tedious task due to the problem of finding the best match of resource-workload pair based on the user QoS requirements. The goal of Cloud workload analyzer is to categorize the workloads and the goal of resource scheduler is to map and schedule the workloads effectively and efficiently. The resources and Cloud workloads can leave and join the Cloud dynamically. Cloud resources are heterogeneous and dynamic in nature. In this work, independent Cloud workloads have been considered to handle the realistic scenarios as there are many scenarios in which the need of scheduling Cloud workloads arises. Firstly, this problem is suitable to Cloud systems because of the nature of Cloud customers, who submit Cloud workloads in an independent manner to the system. Secondly, Cloud systems are most useful for massive parallel processing, in which large amounts of data are processed independently. In this work, the scheduling of workloads has been considered from both the Cloud customer and Cloud provider's point of view. The user wants to minimize the cost whereas the Cloud provider wants to minimize the execution time and energy consumption. In this problem, the most popular and extensively studied optimization criteria, i.e. the minimization of the execution time has been considered. Execution time is used to indicate the general productivity of the Cloud systems. Smaller values of execution time and energy consumption indicate that the scheduler is planning the Cloud workloads in an efficient manner. Cost is another optimization criterion, which refers to the total cost of the Cloud workload execution on a particular resource. The problem has been derived to get an optimal solution. 
The problem can be expressed as: To consider this problem, a set of independent Cloud workloads $\left\{w_{1}, w_{2}, w_{3}, \ldots\right.$ ,$\left.w_{m}\right\}$ to map on a set of heterogeneous and dynamic resources $\left\{r_{1}, r_{2}, r_{3}, \ldots, r_{n}\right\}$ has been taken. For continuous problem, $\mathrm{R}=\left\{r_{k} \mid 1 \leq \mathrm{k} \leq \mathrm{n}\right\}$ is the collection of resources and $n$ is the total number of resources. $\mathrm{W}=\left\{w_{i} \mid 1 \leq \mathrm{i} \leq\right.$ $\mathrm{m}\}$ is the collection of Cloud workloads and $m$ is the total number of Cloud workloads. The estimated time to compute the value of each Cloud workload on each resource is assumed to be given by the consumer-supplied information (data-driven). Under the Predictable Time to Compute (PTC), the following assumptions have been considered:

1. Each Cloud workload to be scheduled for application's execution has a unique workload id.

2. Cloud workloads are independent.

3. Arrival of Cloud workloads for execution of application is random and Cloud workloads are placed in a queue of unscheduled Cloud workloads.

4. The processing speed of the resources is measured in Multiple Instructions Per Second (MIPS) as per the Standard Performance Evaluation Corporation (SPEC) benchmark.

5. The processing requirement of a Cloud workload is measured in Million Instructions (MIs).

6. Execution time for every Cloud workload on a resource is obtained from objective function. [Number of workloads $\times$ number of resources] for every workload on resources is calculated from PTC matrix. Columns of PTC matrix demonstrate the estimated execution time for a specific resource while rows on PTC matrix demonstrate the execution time of a workload on every resource. PTC $\left(w_{i}, r_{k}\right)$ is the expected execution time of workload $w_{i}$ and the resource $r_{k}$.

\subsubsection{Objective function}

In Cloud computing, provider wants to minimize the execution time and energy consumption while user wants to minimize the cost for Cloud workload. The goal of an objective function is to optimize the QoS parameters (execution cost, time and energy consumption) for finishing all $n$ workloads of a given Bulk of Workloads (BoW). This objective function successfully captures the compromise among QoS parameters as specified in Equation (1). Further formally, the workload assignment problem with the energy, cost and time function of each resource $r$ can be generally formulated as follows:

$$
\text { Fitness value }=\alpha \text { execution }_{\text {cost }}+\beta \text { execution }_{\text {time }}+\gamma \text { energy }_{\text {consumption }}
$$

where $0 \leq \alpha<1,0 \leq \beta<1$ and $0 \leq \gamma<1$ are weights to prioritize components of fitness function.

a) Execution Cost $\left(\right.$ execution $_{\text {cost }}$ ): It is the cost spend to execute workload and measured in terms of Cloud Dollars (C\$):

$$
\text { execution }_{\text {cost }}=\min \left(c\left(r_{k}, w_{i}\right)\right) \text { for } 1 \leq k \leq n \text { and } 1 \leq i \leq m
$$

where $c\left(r_{k}, w_{i}\right)$ is the cost of workload $w_{i}$ which executes on resource $r_{k}$ as defined below:

$$
\mathrm{c}\left(r_{k}, w_{i}\right)=\sum_{w_{i} \in W}\left(\frac{\text { completion }\left(w_{i}, r_{k}\right)}{\text { completion }_{m\left(w_{i}\right)} \times W}\right)
$$

$W$ is the collection of cloud workloads.

$$
\mathrm{c}\left(r_{k}, w_{i}\right)=\frac{1}{W} \sum_{w_{i} \in W}\left(\frac{\text { completion }\left(w_{i}, r_{k}\right)}{\text { completion }_{m\left(w_{i}\right)}}\right)
$$

where as:

$$
\begin{gathered}
\text { completion }_{m\left(w_{i}\right)}=\max _{w_{i} \in W, r_{k} \in R} \text { completion }\left(w_{i}, r_{k}\right) \\
\text { execution }_{\text {time }}=\min \left(L_{w_{i}}\right) w_{i} \in W
\end{gathered}
$$




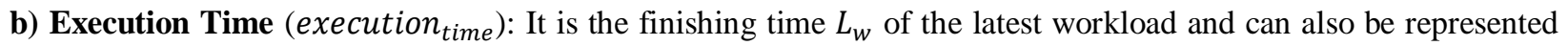
as PTC workload $w_{i}$ on resource $r_{k}$. Before estimation of execution time, completion time of a resource should be defined. Completion time can be defined as the time in which resource can finish the execution of all the previous workloads in addition to the execution of workload $w_{i}$ on resource $r_{k}$ as described as:

$$
\text { completion }\left(r_{k}\right)=\text { available_time } r_{k} \pm P T C_{m\left(w_{i}\right)}
$$

where as:

$$
P T C_{m\left(w_{i}\right)}=\max _{w_{i} \in W, r_{k} \in R} \operatorname{PTC}\left(w_{i}, r_{k}\right)
$$

c) Energy Consumption $(E C)$ : The energy model is devised on the basis that resource utilization has a linear relationship with energy consumption [22]. Energy Consumption of using resources can be expressed as the following formula [Eq. 9]:

$$
E C=E C_{\text {Datacenter }}+E C_{\text {Transceivers }}+E C_{\text {Memory }}+E C_{E x t r a}
$$

$E C_{\text {Datacenter }}$ represents the datacenter's energy consumption, $E C_{\text {Transceivers }}$ represents the energy consumption of all the switching equipment. $E C_{\text {Memory }}$ represents the energy consumption of the storage device. $E C_{E x t r a}$ represents the energy consumption of other parts, including the fans, the current conversion loss and others. $E C_{t, i}$ is the energy consumption at given time $t$ is defined in [Eq. 10]:

$$
E C_{t, i}(r)=q \times E C_{\max }+(1-q) \times E C_{\max } \times r u
$$

Where $E C_{\max }$ is maximum energy consumption while resource is fully utilized, $q$ is fraction of energy consumed by idle resource and $r u$ is resource utilization. Resource utilization is change over time and it is function of time and presented as $r u(t)$. For a resource $r_{t}$ at given time $t$, the resource utilization $\operatorname{Res} U_{t}$ is defined as [Eq. 11]:

$$
\operatorname{Res} U_{t}=\sum_{i=1}^{n} E C_{t, i}(r u(t)) d t
$$

where $n$ is the number of cloud workloads running at time $t$. The actual energy consumption $E C_{\text {actual }}$ of a resource $r u_{t}$ at given time $t$ is defined as [Eq. 12]:

$$
E C_{\text {actual }}=\left(E C_{\max }-E C_{\min }\right) \times R e s U_{t}+E C_{\min }
$$

where $E C_{\max }$ is the energy consumption at the peak load (or $100 \%$ utilization) and $E C_{\min }$ is the minimum energy consumption in the active/idle mode (or as low as $1 \%$ utilization).

\subsubsection{Particle Swarm Optimization}

Particle Swarm Optimization (PSO) is a group based intelligence algorithm which is inspired by the social behavior such as school of fish defending themselves from a hunter (predator) or group of birds finding a source of food [19]. Population in PSO algorithm is defined as the total number of particles in a problem space and particles in population are initialized randomly. In every generation, fitness value of every particle is estimated by a fitness function to be improved. Both the positions of the particle is known: i) best position (local best i.e. $L_{B P}$ ) of a particle and global best (the best position so far among the whole group of particles $\left(G_{B P}\right)$ ). $G_{B P}$ is s the best particle in terms of fitness in an whole population, whereas $L_{B P}$ of a particle is the best result (fitness value) so far reached by the particle. Particle's position and velocity is updated in every generation by using [Eq. 1].

PSO optimization technique which works based on global search. There is no straight re-combination of individuals of the population in algorithm of PSO like other population-based algorithms (or evolutionary algorithms) such as GA (Genetic Algorithm) etc. Algorithm of PSO is dependent on particle's social behavior. Every individual particle regulates its path based on the position of the best particle (global best) of the whole population and its best position 
(local best) in every generation. Stochastic nature of the particle increases due to this property of PSO and touches rapidly to global minima with a realistic noble solution [19]. PSO has become prevalent due to its easiness and its usefulness in extensive range of application with little cost of computation. Applications like pattern recognition, reactive data mining etc. have used PSO mostly. Along with these applications PSO has been solved various NPHard problems like workload allocation and resource scheduling.

\subsubsection{PSO Terminology}

The PSO terminology used in this research paper is described below:

a) Particle: A particle in Particle Swarm Optimization is similar to a fish or bird flying through a search space (problem area). Every particle's movement is synchronized by a velocity which has both direction and magnitude. Position of every particle at any time instance is influenced by its best position $\left(L_{B P}\right)$ and the position of the best particle $\left(G_{B P}\right)$ in a search space. Fitness value is used to measure the performance of a particle, which is problem oriented. For this research work, a workload is considered as a particle.

b) Population Size: In this research work, size of set of workloads (number of workloads considered as a population size).

c) Random Velocity: Every particle's movement is the composition of an initial random velocity and two randomly weighted effects: i) the affinity to return to the best previous position of particle and ii) the affinity to move towards the best previous position of neighborhood. Based on these two affinities of workload, the mapping of workload with resources is done. Workload will be executed only with that resource which has high value of fitness.

d) Particle Velocity: It is calculated based on the probability distribution for the particle position, that is, the particle (workload) position in a dimension is randomly generated using that distribution.

e) Particle Position: Current state of particle (workload), state may be submission state, waiting state, ready state, execution state and completion state.

f) Global Best Position $\left(G_{B P}\right)$ : Best position of particle (workload) among the whole group of particles (set of workloads).

g) Local Best Position $\left(L_{B P}\right.$ ): Best position of particle (workload) as reached by the particle (workload regulates its path based on its best resource which executes workload with minimum fitness value).

\subsubsection{PSO Based Resource Scheduling Algorithm}

In this section, we present the pseudo code of PSO-based algorithm for resource scheduling in the Cloud environment. Each particle in genome is a partial solution and is represented as a resource identifier (e.g., select, move, swap, drop) or a sequence of resource identifiers. The non-PSO based resource identifier can be simple or complex and are implemented as follows:

a. Workload selection and scheduling: the resource identifiers select workload from the unscheduled list and schedule it in to the best available resource.

b. Try for the best combination of all workloads and resources until the best combination is found.

c. Move workload $\left(w_{i}\right)$ from its current resource/schedule.

d. Swap workloads: select the workloads randomly which can swap.

e. Remove a randomly selected workload from workload queue already scheduled.

This is the only heuristic which will move the search into an infeasible region because any workload may be unscheduled. We make sure that the search can move back into its feasible region by un-scheduling workload that has other valid resources so that it can move into the next iteration. The non-PSO based resource identifier is then applied so as to find an optimal solution of the problem instance. The objective of PSO is to find the best resource identifier that generates the best solution for resource scheduling problem. The selection process of non-PSO based resource identifier stops after a pre-defined number of iterations. We set a fixed number of iterations to keep the computation time low. The particle rejects the new solution if it is poorer than the current solution. The pseudo code of PSO based resource scheduling algorithm in Figure 2. 


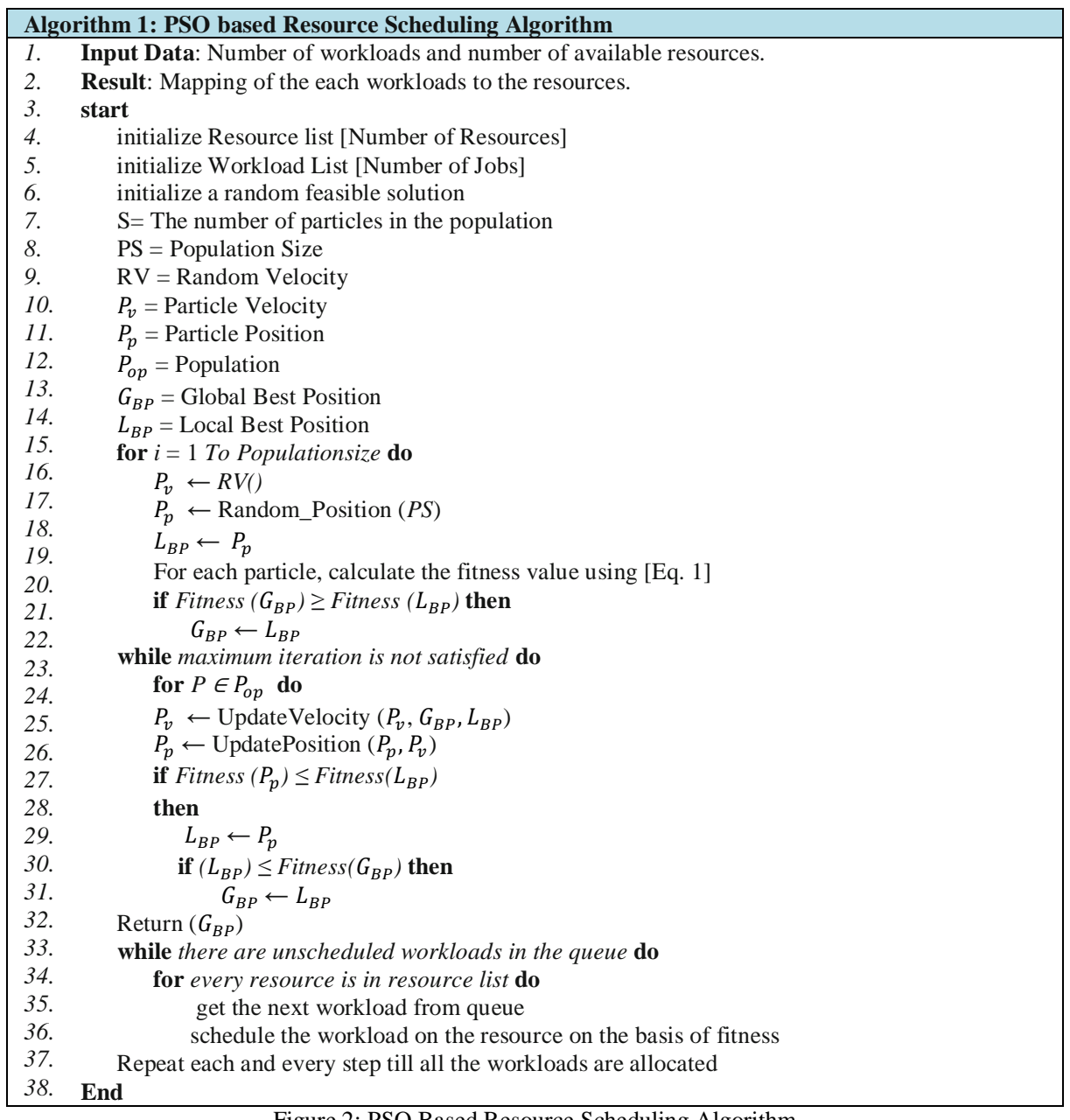

Figure 2: PSO Based Resource Scheduling Algorithm

$>$ A resource list is then obtained from the resource provisioning unit after provisioning of user's workloads [5]. Once the resource list has been obtained, a workload list and a random feasible solution are initialized.

$>$ The task to choose the best heuristic from low-level heuristics is started.

$>$ We have a number of workloads, each of which represents a resource identifier supplied with an initial solution in the solution space and an access to the evaluation function.

$>$ Workload's position and Workload's velocity would be randomly initialized.

$>$ It will then select a low-level heuristic at each workload position and compute its fitness function i.e. Fitness $\left(L_{B P}\right)$.

$>$ If at $P_{p}$, Fitness $\left(L_{B P}\right)$ is better than Fitness $\left(G_{B P}\right)$ then $G_{B P}$ takes the value of $L_{B P}$.

$>$ We will try to find the Fitness value at best global position of the workload.

$>$ After a workload has been chosen from the population, its position and velocity would be updated using [Eq. 1]. Then, its fitness at the new position is calculated and compared with its previous position.

$>$ If it is better than the local best value then we will assign workload's current position to the local best value.

$>$ Now, we will compare fitness at $L_{B P}$ and $G_{B P}$. If the fitness at $L_{B P}$ is better than at $G_{B P}$ then we will assign the value of $L_{B P}$ to $G_{B P}$.

$>$ After selection of a low-level heuristic, it is then applied to the problem. Resource scheduling is performed till there are no unscheduled jobs in the queue. 


\subsection{Cloud Workload Execution Design}

There are eight different classes created to represent the interaction among different workload related entities. Workloads are scheduled to the appropriate resources based on the workload description by taking care of QoS. Fitness value for every workload is calculated and analysed. Schedule the resources to cloud workloads and execute within the defined budget and desired deadline with minimum energy consumption. The interaction among different classes for workload execution is shown in Figure 3. In the course of its lifetime, a workload passes through many states as is outlined in Figure 4. A workload is an input to the scheduler which allocates it to a set of provisioned resources based on its requirements. The workload's status is then changed to SCHEDULED. During the STAGE_IN state, input files and executables required for the workload are staged to the available provisioned resource. When this process is completed successfully, then a workload is considered to be SUBMITTED. The workload may be queued while waiting for an available processor and its state changes to PENDING. When the workload starts its execution, it is considered ACTIVE. After the workload has finished executing, it enters the STAGE_OUT stage where its output files are transferred back to the broker. If all its outputs are received and are as expected by the task requirements, then the workload is considered as "DONE". If one of state transition fails on the available side or the workload is completed on the available side but has not produced the expected result files, then it is considered FAILED and is reset and marked for re-scheduling.

\subsection{QoS Metrics}

The following other metrics ([Eq. 13] - [Eq. 18]) are selected from our previous work [5] [6] [23] to measure the value of QoS parameters other than energy, cost and time.

Availability (A): It is a ratio of Mean Time Between Failure (MTBF) to addition of Mean Time Between Failure (MTBF) and Mean Time To Repair (MTTR). We have used following formula to calculate availability [Eq. 13].

$$
A=\frac{M T B F}{M T B F+M T T R}
$$

Where Mean Time Between Failure (MTBF) is ratio of total uptime to number of breakdowns [Eq. 14].

$$
M T B F=\frac{\text { Total Uptime }}{\text { Number of Breakdowns }}
$$

Where Mean Time To Repair (MTTR) is ratio of total downtime to number of breakdowns [Eq. 15].

$$
M T T R=\frac{\text { Total Downtime }}{\text { Number of Breakdowns }}
$$

Reliability (re): Reliability of the resource has to be checked for scheduling of the resources. With the help of reliability parameter, we can check the fault tolerance of the resource. Reliability of the resource is calculated with the following formula [Eq. 16] as:

$$
r e=e^{-\lambda \mathrm{t}}
$$

$r e=$ reliability of resource, $e=$ exponential function, $t=$ time for resource to deal with its request for any workload's execution and $\lambda=$ the failure rate of the resource at the give time.

Resource Utilization (RU): It is a ratio of execution time of a workload executed by a particular resource to total uptime of that resource. We have used following formula to calculate resource utilization [Eq. 17].

$$
\mathrm{RU}=\sum_{i=1}^{n}\left(\frac{\text { execution time of a workload executed } i^{\text {th }} \text { resource }}{\text { total uptime of } i^{\text {th }} \text { resource }}\right)
$$


Where $n$ is number of workloads.

Latency ( $\mathbf{L})$ : It is defined as a difference between expected execution time and actual execution time. We have used following formula to calculate Latency [Eq. 18]:

$$
\mathrm{L}=\sum_{i=1}^{n}\left(\text { Expected Execution } \text { Time }_{i}-\text { Actual Execution Time } \text { Tim }_{i}\right)
$$

Where $n$ is number of workloads.

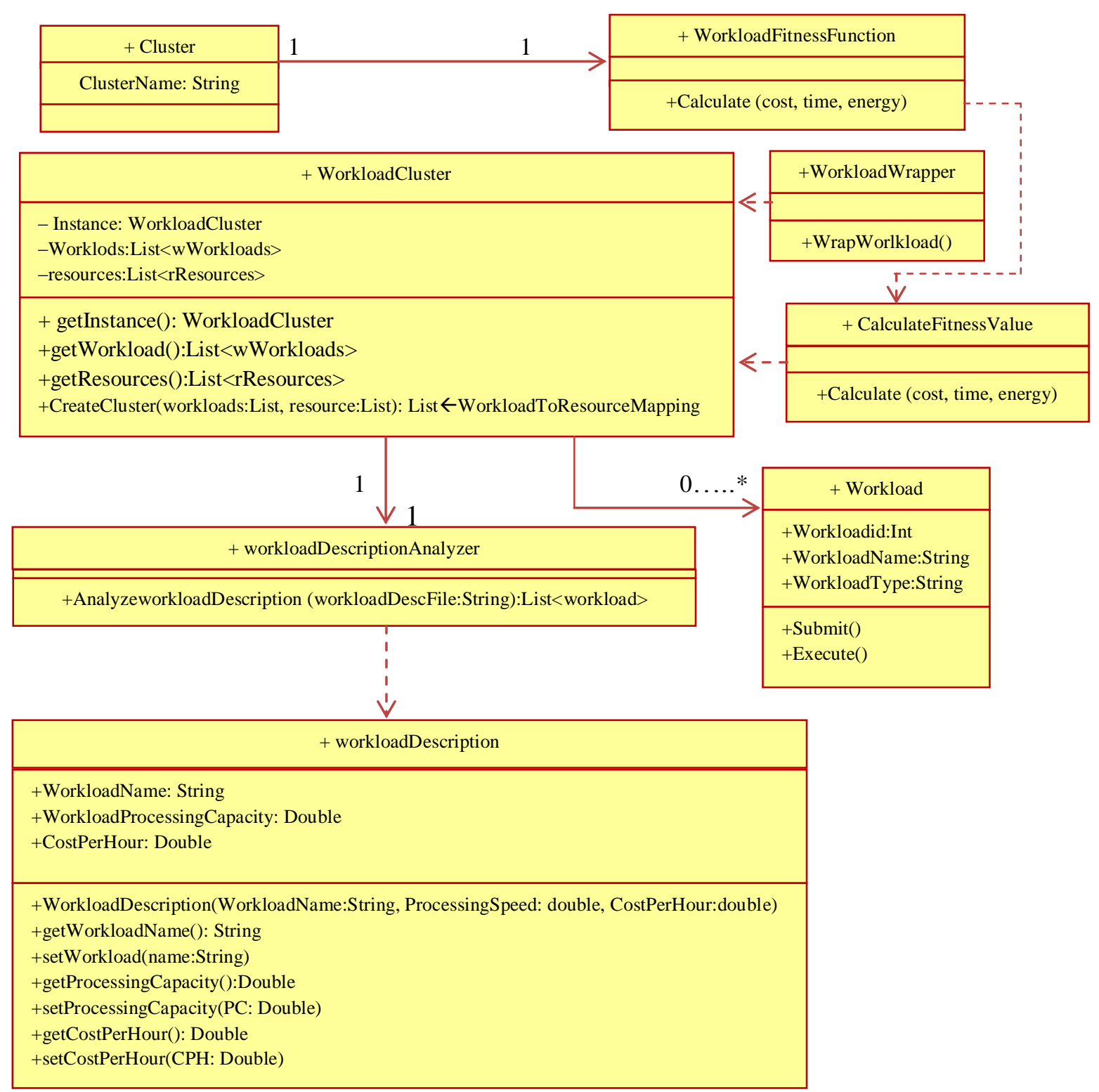

Figure 3: Interaction Among Different Classes For Workload Execution 


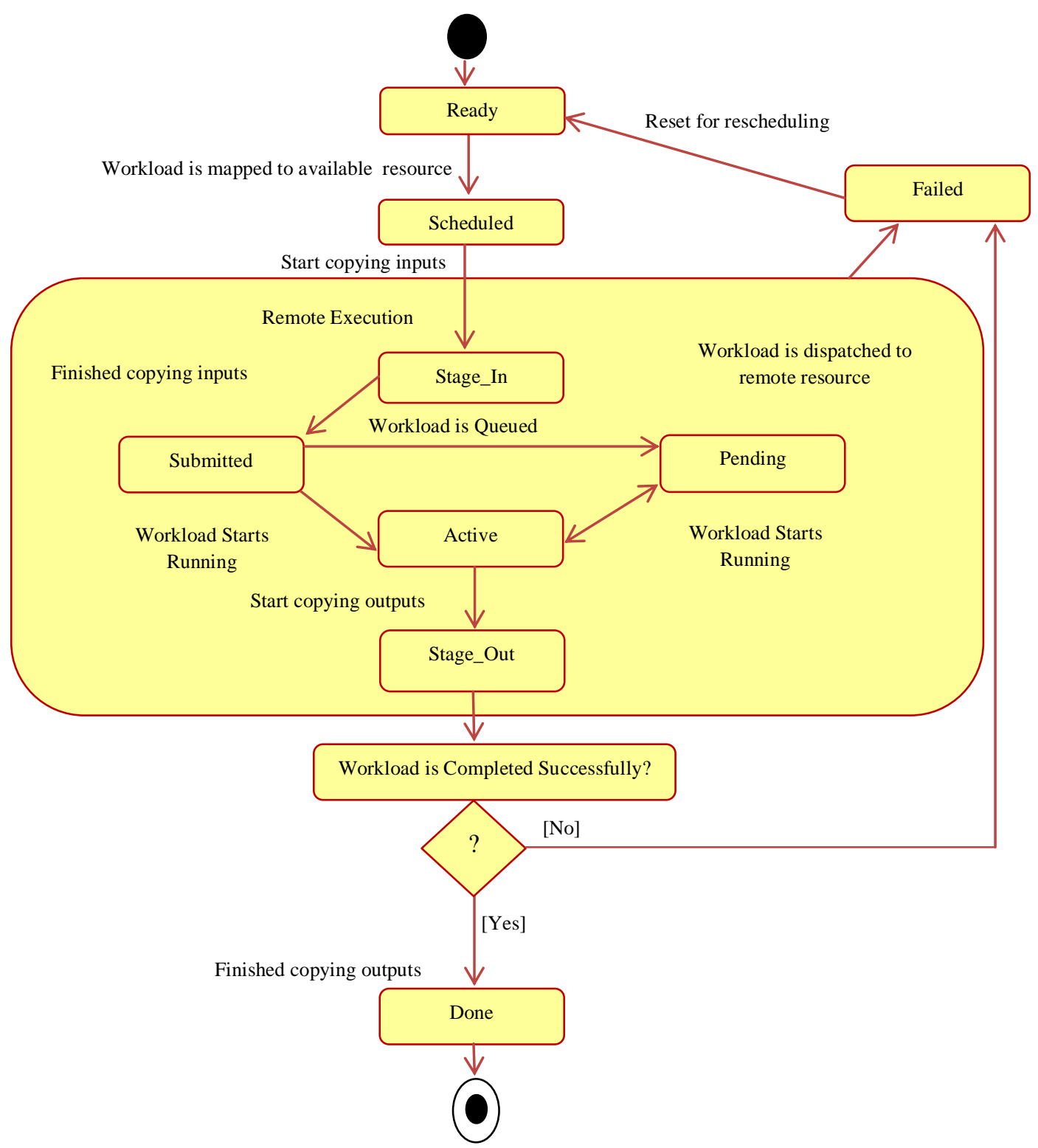

Figure 4: Different Stages For Cloud Workload

\section{Experimental Setup and Results}

Tools used for setting up cloud environment for performance analysis are Microsoft Visual Studio 2010 (SaaS), Aneka (PaaS), SQL Server 2008, and Citrix Xen Server (IaaS). Aneka has been installed along with its requirements on all the nodes that provide cloud service. Nodes in this system can be added or removed based on the requirement. PSO based resource scheduling technique (BULLET) is installed on main server and tested on virtual cloud environment that has been established at CLOUDS Lab, The University of Melbourne, Australia. We installed different number of virtual machines on different servers, and deployed the BULLET to measure the variations. In this experimental setup, three different cloud platforms are used: Software as a Service (SaaS), Platform as a Service (PaaS) and Infrastructure as a Service (IaaS) as shown in Figure 5. 


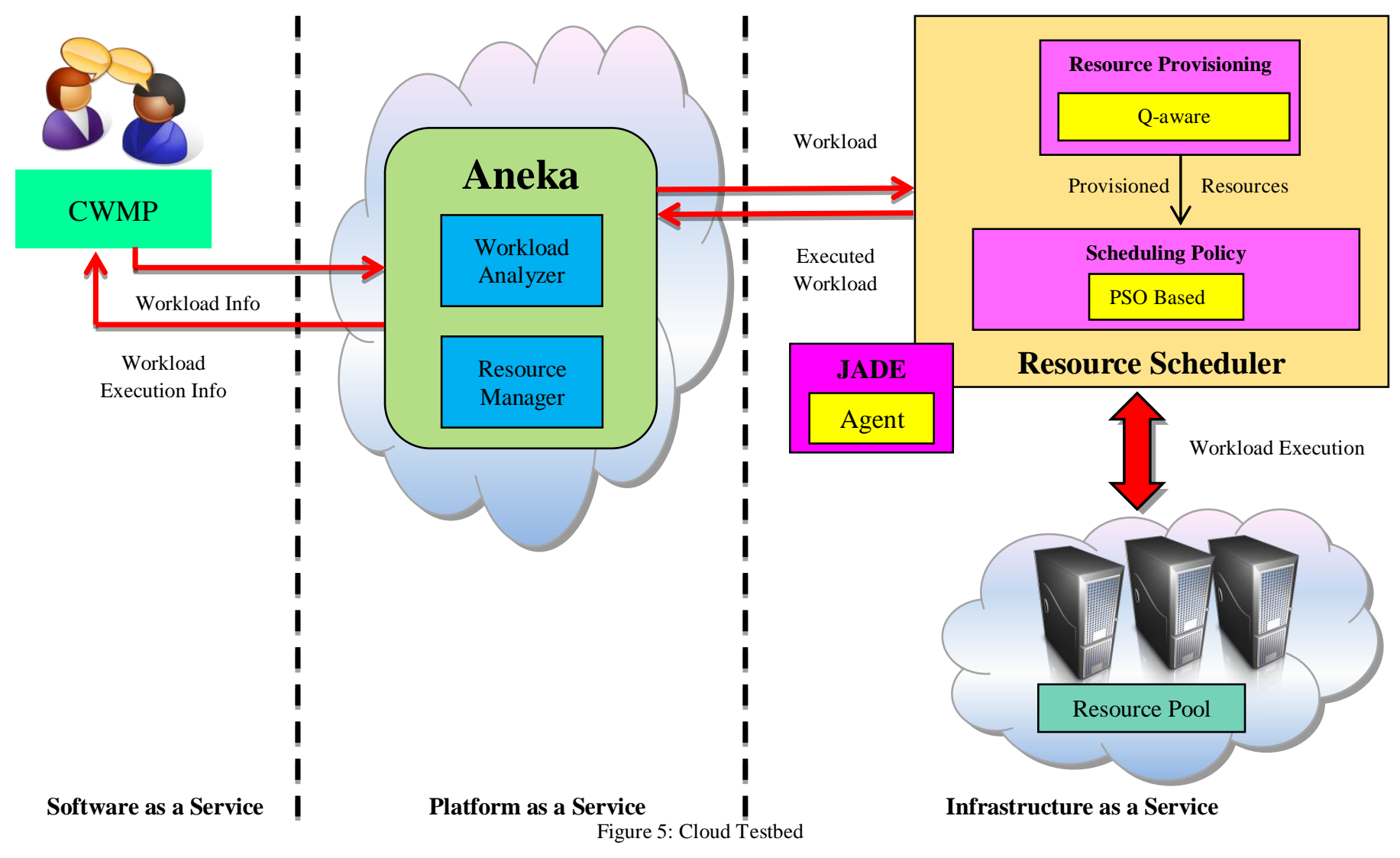

At SaaS level, Microsoft Visual Studio 2010 is used to develop Cloud Workload Management Portal (CWMP) to provide user interface in which user can access service from any geographical location. At PaaS level, Aneka cloud application platform [19] [23] is used as a scalable cloud middleware to make interaction between IaaS and SaaS, and continually monitor the performance of the system. Aneka task model has been used in this research work. A task is a single unit of work processed (request) in a node. It is independent from other tasks that may be executed on the same or any other node at the same time. At IaaS level, three different servers (consist of virtual nodes) have been created through Citrix Xen Server and SQL Server has been used for data storage. Scheduler as shown in Figure 5, runs at IaaS level on Citrix Xen Server. Computing nodes used in this experiment work are further categorized into three categories as shown in Table 4. A detailed discussion of the implementation using Aneka can be found in [23].

Table 4: Configuration Details

\begin{tabular}{|c|c|c|c|c|c|c|}
\hline Resource_Id & Configuration & Specifications & $\begin{array}{c}\text { Operating } \\
\text { System }\end{array}$ & $\begin{array}{c}\text { Number of } \\
\text { Virtual Node }\end{array}$ & $\begin{array}{c}\text { Number of } \\
\text { ECs }\end{array}$ & $\begin{array}{c}\text { Price } \\
\text { (C\$/EC time } \\
\text { unit) }\end{array}$ \\
\hline R1 & Intel Core 2 Duo - $2.4 \mathrm{GHz}$ & 1 GB RAM and 160 GB HDD & Windows & 6 & 18 & 2 \\
\hline $\mathrm{R} 2$ & Intel Core i5-2310- $2.9 \mathrm{GHz}$ & 1 GB RAM and 160 GB HDD & Linux & 4 & 12 & 3 \\
\hline R3 & Intel XEON E 52407-2.2 GHz & 2 GB RAM and 320 GB HDD & Linux & 2 & 6 & 4 \\
\hline
\end{tabular}

The execution cost is calculated based on user workload and deadline (if deadline is too early (urgent) it will be more costly because we need a greater processing speed and free resources to process particular workload with urgency. There individual price is fixed (artificially) for different resources because all the resources are working in coordination manner to fulfill the demand of user (demand of user is changing dynamically). Experiment setup using 3 servers in which further virtual nodes $(12=6$ (Server 1$)+4($ Server 2$)+2($ Server 3$))$ are created. Every virtual node has different number of Execution Components (ECs) to process user workload and every EC has their own cost $(\mathrm{C} \$ / \mathrm{EC}$ time unit $(\mathrm{Sec}))$. Table 4 shows the characteristics of the resources used and their Execution 
Component (EC) access cost per time unit in Cloud Dollars (C\$) and access cost in C $\$$ is manually assigned for experimental purposes. The access cost of an $\mathrm{EC}$ in $\mathrm{C} \$ /$ time unit does not necessarily reflect the cost of execution when ECs have different capabilities. The execution agent needs to translate the access cost into the C\$ for each resource. Such translation helps in identifying the relative cost of resources for executing user workloads on them.

Due to limited number of resources, cost increases with increase in user workloads. Cost is varying in two different cases: i) relaxed deadline and ii) tight deadline. In both cases, when the deadline is low (e.g. 200 secs), the number of user workloads processed increases as the budget value increases. When a higher budget is available, the execution agent uses expensive resources to process more user workloads within the deadline. Alternatively, when scheduling with a low budget, the number of user workloads processed increases as the deadline is relaxed. Execution agent allocates as many user requests as the first cheapest resource can complete by the deadline, and then allocates the remaining user workloads to the next cheapest resources. When the deadline is tight (e.g. 100), there is high demand for all the resources in a short time. All the resources are used up so long as budget is available to process all user workloads within the deadline. However, when the deadline is relaxed (e.g. 700 secs), it is likely that all user workloads can be completed using the first few cheapest resources. As the deadline increases, execution agent schedules user workloads on the available resources to finish earlier as possible. The aim of this performance evaluation is to demonstrate that it is feasible to implement and deploy the proposed technique on real cloud resources. The key components of the cloud environment are: user interface (CWMP), workload analyzer and resource scheduler. Figure 6 enables the understanding of the cloud based environment in which the proposed technique is implemented.

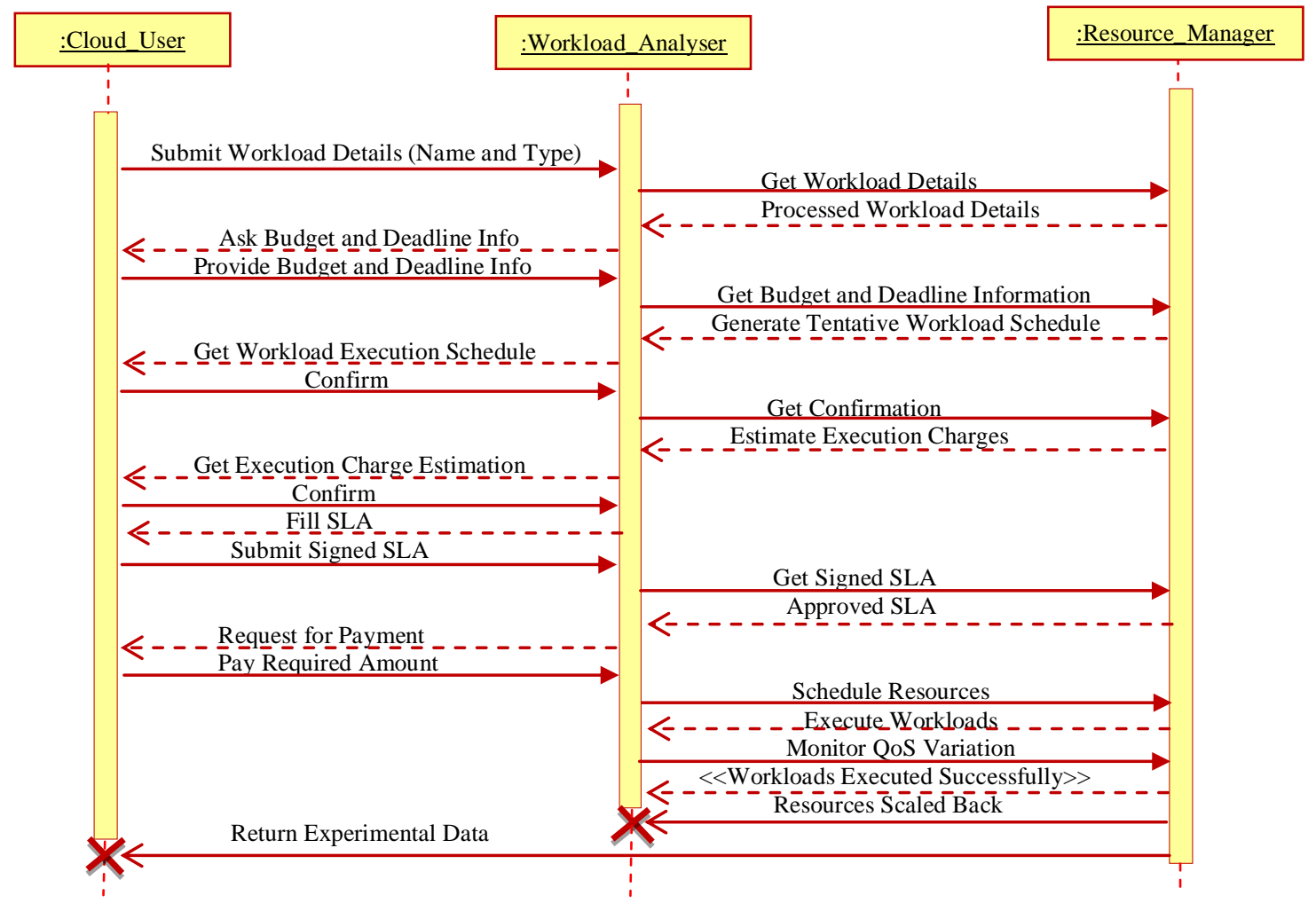

Figure 6: Workload Execution

\subsection{Performance Evaluation}

In order to evaluate the performance of BULLET, we have compared the value of QoS parameters (execution time, cost, energy consumption, reliability, availability, latency and resource utilization) of BULLET with existing PSO based cloud based resource scheduling techniques (PSO-HPC [14], PSO-SW [15] and PSO-DVFS [16]) and all the three existing techniques have been described in Section 2.2. Formals used to measure the value of QoS parameters have been described in Section 3. We have performed experiments to determine the effect of an increase in number 
of workloads and resources on QoS parameters such as energy consumption, cost and time. All the experiments were started with workload name: Performance Testing [Processing Larger Image File of Size 713 MB], in which BULLET converts an image file from JPEG format to PNG format. Conversion of a single JPEG file into PNG is considered as a single workload. We have created the PTC matrix which is computed as ratio of workload and computing capacity of virtual machines.

\subsection{Experimental Results}

Experiment has been conducted with different number of cloud workloads (15-90) for verification of QoS parameters.

\section{Test Case 1: Energy Consumption vs. Number of Workloads}

By increasing the number of cloud workloads, the value of energy consumption is increasing. The minimum value of energy consumption is $69 \mathrm{kWh}$ at 15 cloud workloads for BULLET. BULLET performs better than PSO-HPC, PSO-SW and PSO-DVFS in terms of energy consumption at different number of cloud workloads as shown in Figure 7. The average value of energy consumption in BULLET is $7.61 \%, 11.45 \%$ and $17.19 \%$ lesser than PSOHPC, PSO-SW and PSO-DVFS respectively.

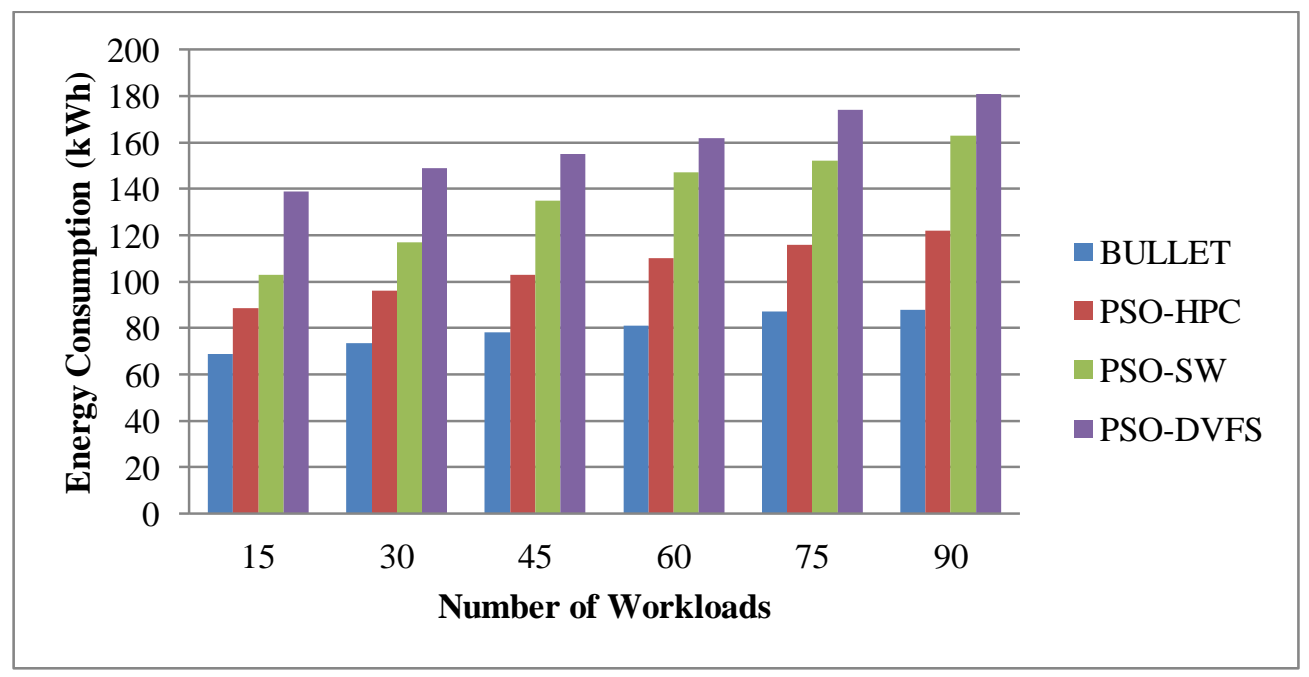

Figure 7: Effect of Change in Number of Workloads Submitted on Energy Consumption

\section{Test Case 2: Execution Cost vs. Number of Workloads}

With the increase in number of workloads, execution cost rises as shown in Figure 8. As per the number of workloads increases, BULLET performs better than PSO-HPC, PSO-SW and PSO-DVFS. BULLET outperforms as it adjusts the resources at runtime according to the QoS requirements of workload. The minimum cost used in BULLET is $171 \mathrm{C} \$$ at 15 workloads and maximum is $416 \mathrm{C} \$$ at 90 workloads. The average value of execution cost in BULLET is 3.16\%, 4.72\% and 9.16\% lesser than PSO-HPC, PSO-SW and PSO-DVFS respectively. 


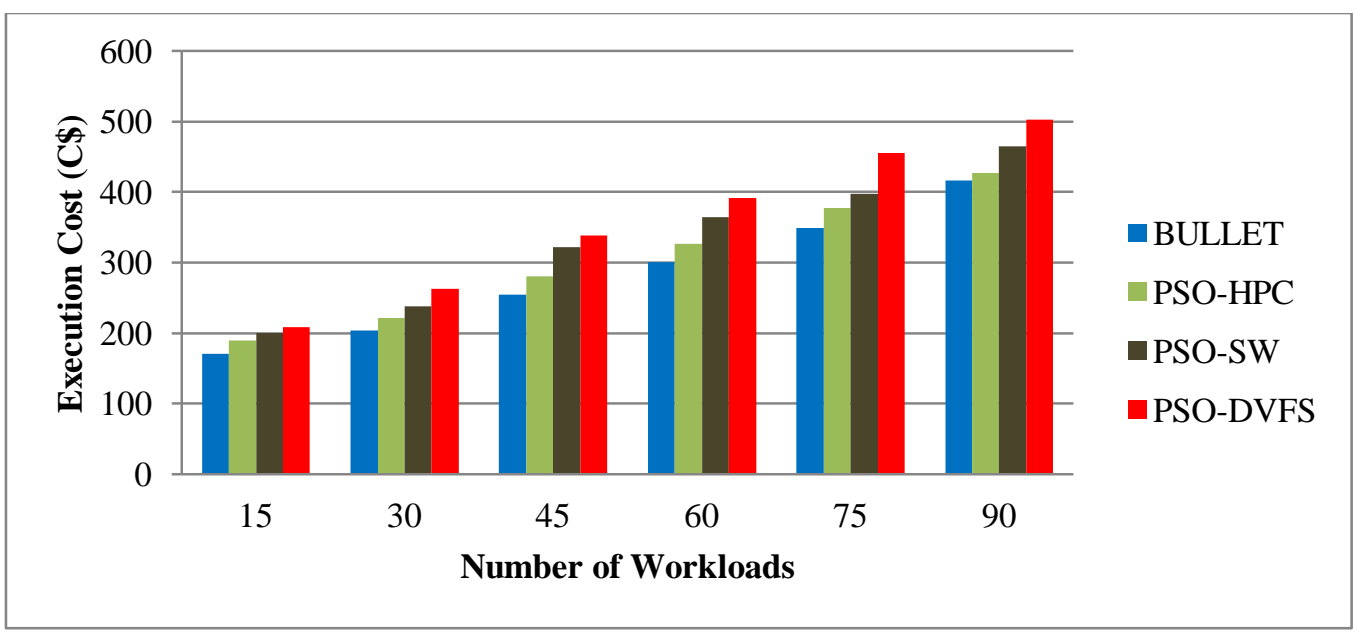

Figure 8: Effect of Execution Cost with Change in Number of Workloads

\section{Test Case 3: Execution Time vs. Number of Workloads}

As shown in Figure 9, the execution time increases with increase in number of workloads. At 30 workloads, execution time in BULLET is 6.69\% lesser than PSO-HPC, 7.12\% lesser than PSO-SW and 7.59\% lesser than PSODVFS. At 90 workloads, execution time in BULLET is $8.72 \%$ lesser than PSO-HPC, $11.39 \%$ lesser than PSO-SW and $14.79 \%$ lesser than PSO-DVFS. Figure 9 show that execution time varies in same ratio but BULLET performs better than PSO-HPC, PSO-SW and PSO-DVFS.

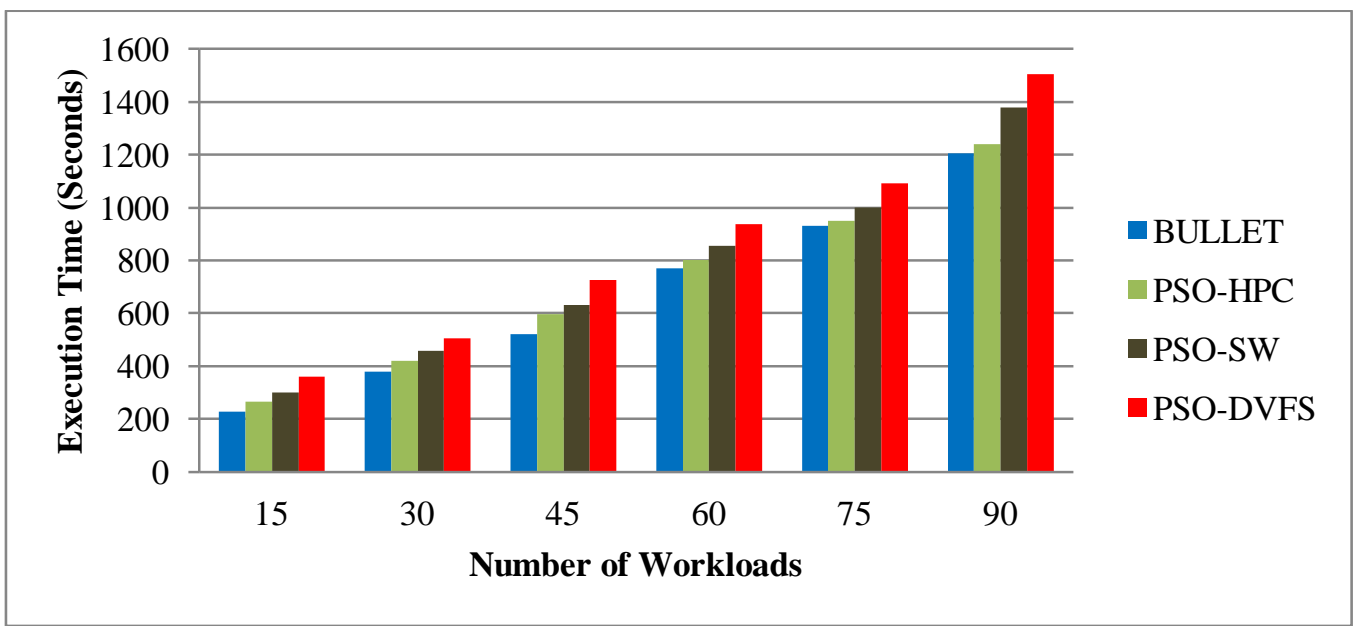

Figure 9: Effect of Execution Time with Change in Number of Workloads

The number of workloads considered for Test Case 4, Test Case 5 and Test Case 6 is 90 .

\section{Test Case 4: Energy Consumption vs. Number of Resources}

By increasing the number of resources, the value of energy consumption increases. The minimum value of energy consumption is $22 \mathrm{kWh}$ at 6 resources for BULLET. BULLET performs better than PSO-HPC, PSO-SW and PSODVFS in terms of energy consumption at different number of resources as shown in Figure 10. The average value of energy consumption in BULLET is $4.41 \%, 8.54 \%$ and $12.36 \%$ lesser than PSO-HPC, PSO-SW and PSO-DVFS respectively. 


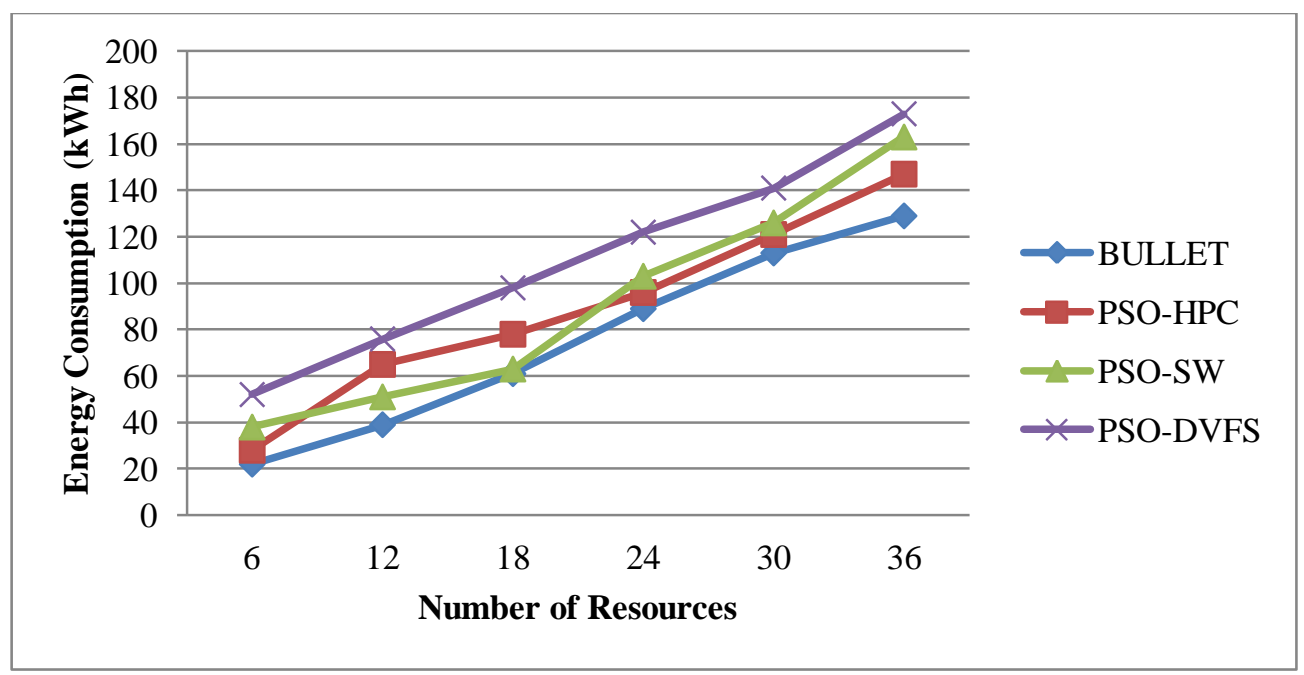

Figure 10: Effect of Change in Number of Resources Submitted on Energy Consumption

\section{Test Case 5: Execution Cost vs. Number of Resources}

With the increase in number of resources, execution cost rises as shown in Figure 11. As per the number of resources increases, BULLET performs better than PSO-HPC, PSO-SW and PSO-DVFS. The cause is that BULLET adjusts the resources at runtime according to the QoS requirements of workload. The minimum cost is used in BULLET is $45 \mathrm{C} \$$ at 6 resources and maximum is $137 \mathrm{C} \$$ at 36 resources. The average value of execution cost in BULLET is $5.41 \%, 6.26 \%$ and $8.78 \%$ lesser than PSO-HPC, PSO-SW and PSO-DVFS respectively.

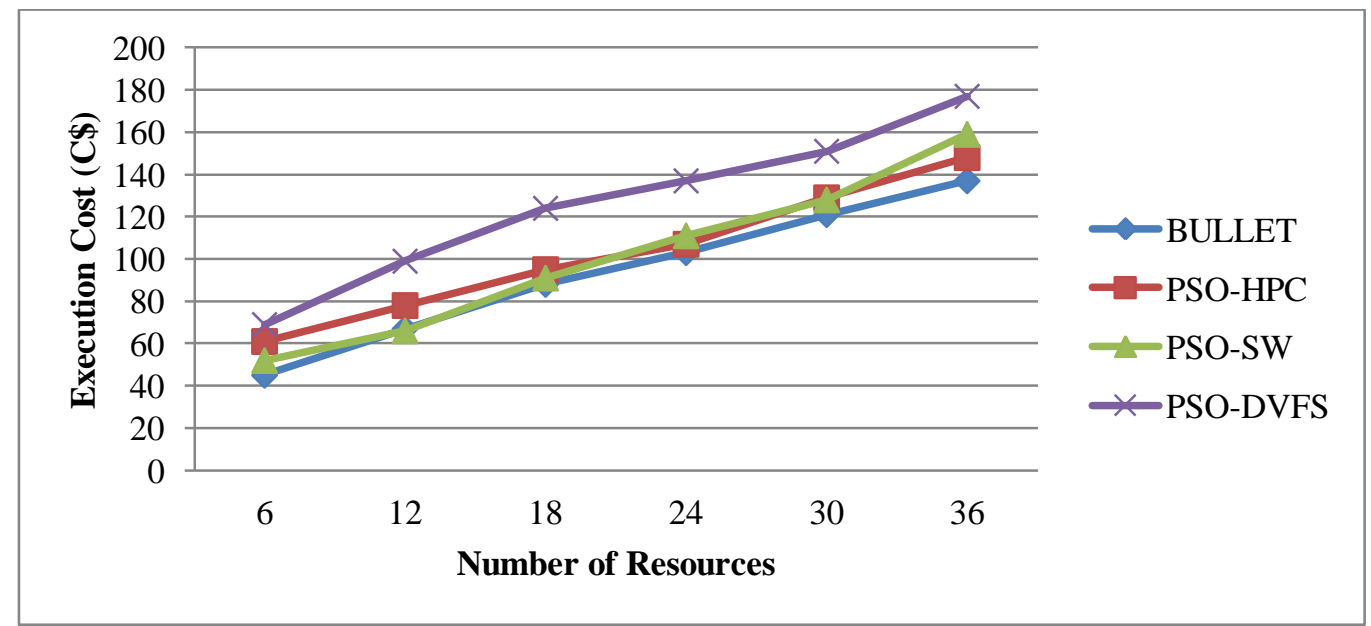

Figure 11: Effect of Execution Cost with Change in Number of Resources

Test Case 6: Execution Time vs. Number of Resources

As shown in Figure 12, the execution time decreases with increase in number of resources. At 18 resources, execution time in BULLET is 7.42\% lesser than PSO-HPC, $8.91 \%$ lesser than PSO-SW and $12.52 \%$ lesser than PSO-DVFS. At 36 resources, execution time in BULLET is 3.49\% lesser than PSO-HPC, $4.64 \%$ lesser than PSOSW and 7.93\% lesser than PSO-DVFS. Figure 12 show that execution time varies in same ratio but BULLET performs better than PSO-HPC, PSO-SW and PSO-DVFS. 


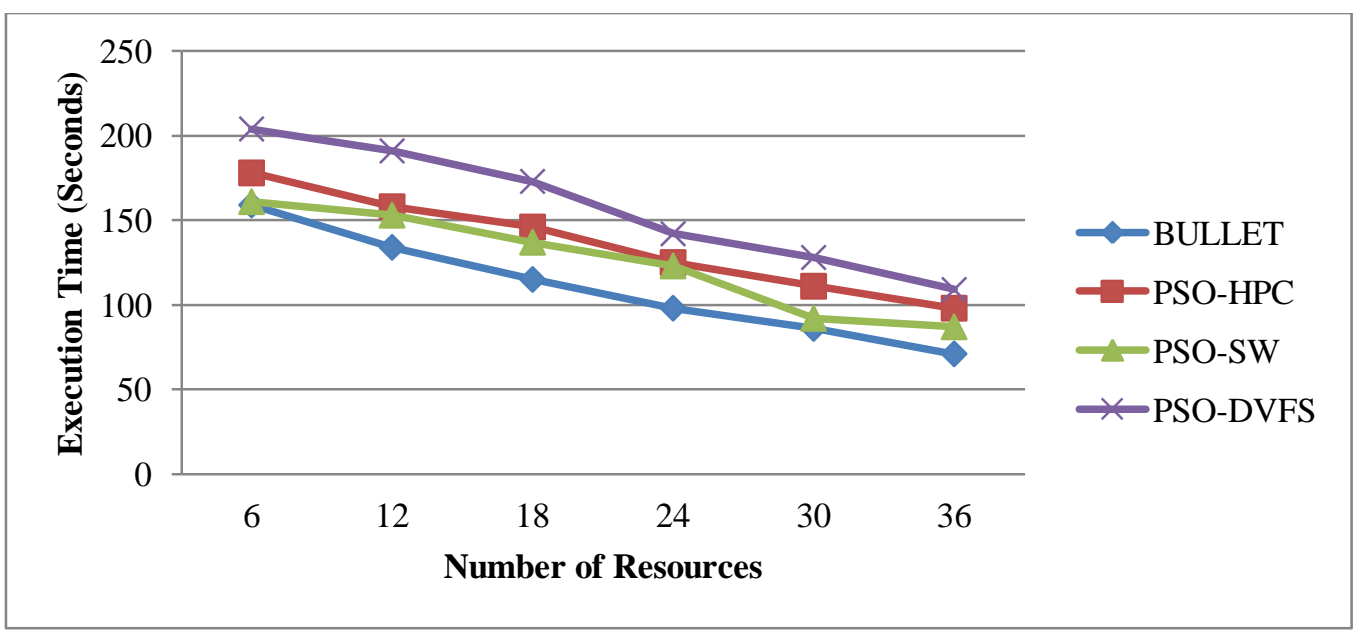

Figure 12: Effect of Execution Time with Change in Number of Resources

\section{Test Case 7: Availability}

We have calculated the percentage of availability for BULLET and existing scheduling algorithms (PSO-HPC, PSOSW and PSO-DVFS) with different number of cloud workloads. With increasing the number of cloud workloads, the percentage of availability is decreasing. The percentage of availability in BULLET is more as compared to PSOHPC, PSO-SW and PSO-DVFS at different number of cloud workloads as shown in Figure 13. The maximum percentage of availability is $88.7 \%$ at minimum number of cloud workloads. At 75 workloads, percentage of availability in BULLET is $7.42 \%$ more than PSO-HPC, 9.91\% more than PSO-SW and $13.72 \%$ more than PSODVFS.

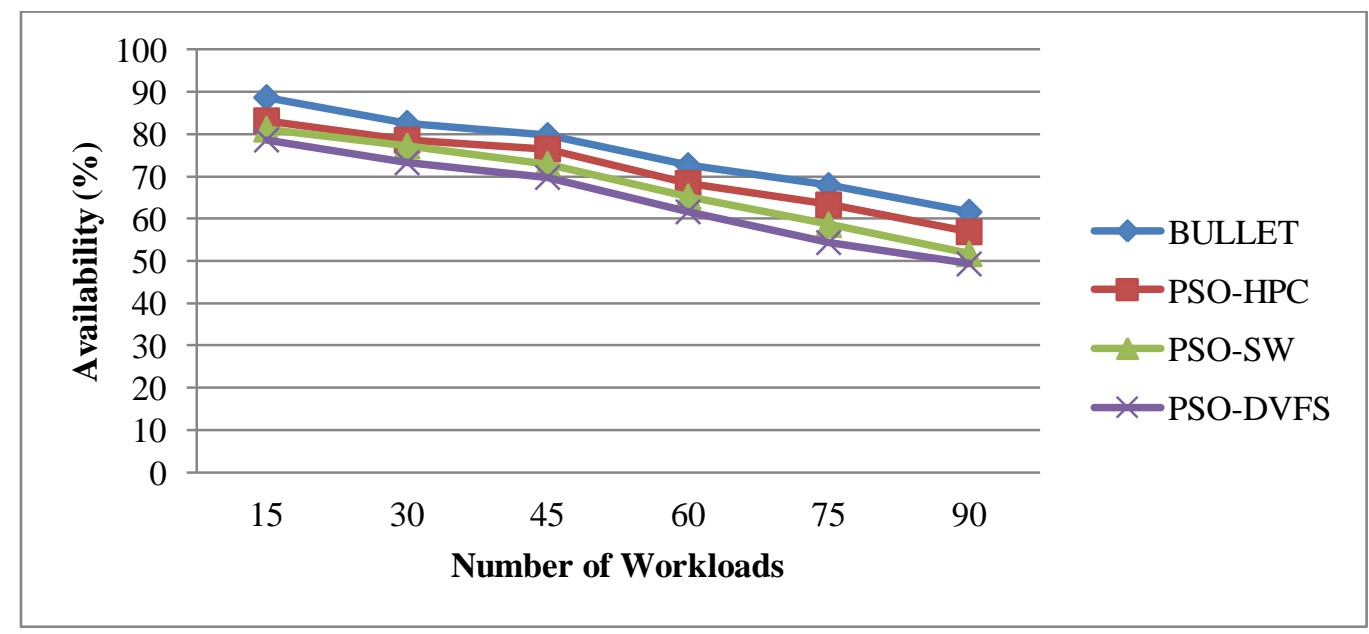

Figure 13: Effect of Change in Number of Workloads Submitted on Availability

\section{Test Case 8: Reliability}

We have calculated the percentage of reliability for BULLET and existing scheduling algorithms (PSO-HPC, PSOSW and PSO-DVFS) with different number of cloud workloads. By increasing the number of cloud workloads, the percentage of reliability is decreasing. The percentage of reliability in BULLET is more as compared to PSO-HPC, PSO-SW and PSO-DVFS at different number of cloud workloads as shown in Figure 14. The maximum percentage of reliability is 94.65 at 15 cloud workloads. At 60 workloads, percentage of reliability in BULLET is $4.91 \%$ more than PSO-HPC, 8.76\% more than PSO-SW and $17.22 \%$ more than PSO-DVFS. 


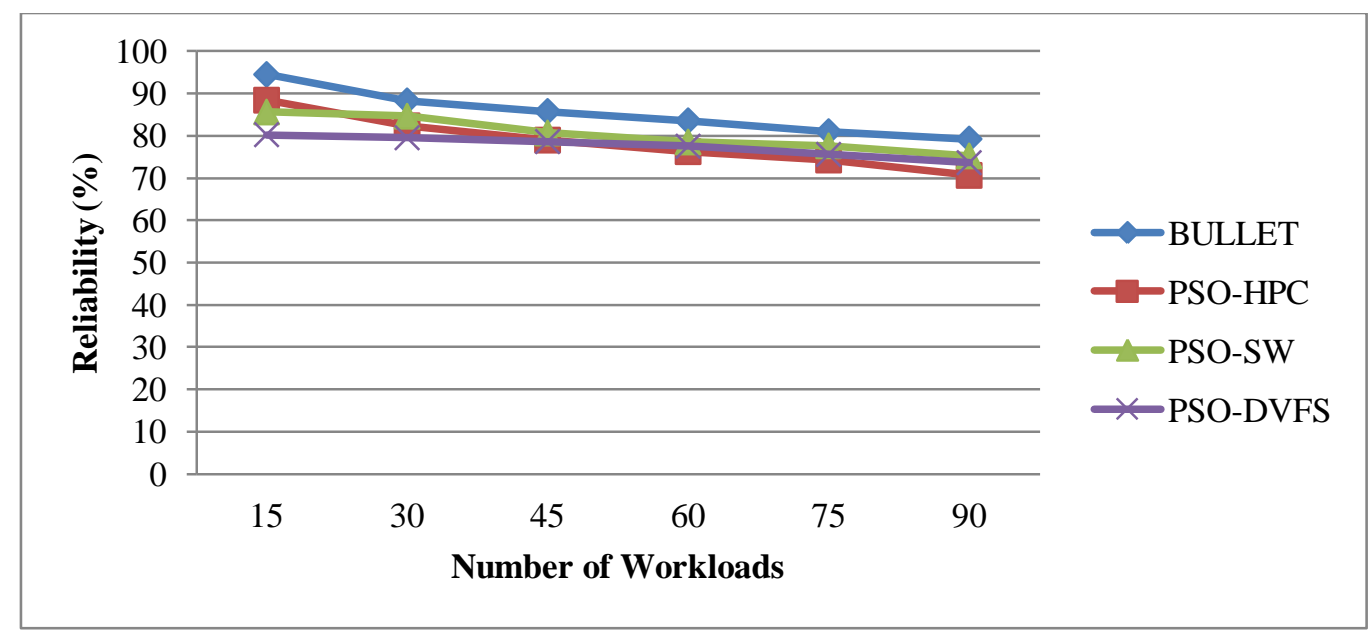

Figure 14: Effect of Change in Number of Workloads Submitted on Reliability

\section{Test Case 9: Resource Utilization}

With increasing number of cloud workloads, the percentage of resource utilization is increasing. The percentage of resource utilization in BULLET is more as compared to PSO-HPC, PSO-SW and PSO-DVFS at different number of cloud workloads as shown in Figure 15. The maximum percentage of resource utilization is $88.4 \%$ at 90 cloud workloads and minimum percentage is 71.96 at 15 workloads in BULLET but BULLET performs better than PSOHPC, PSO-SW and PSO-DVFS for any number of workloads.

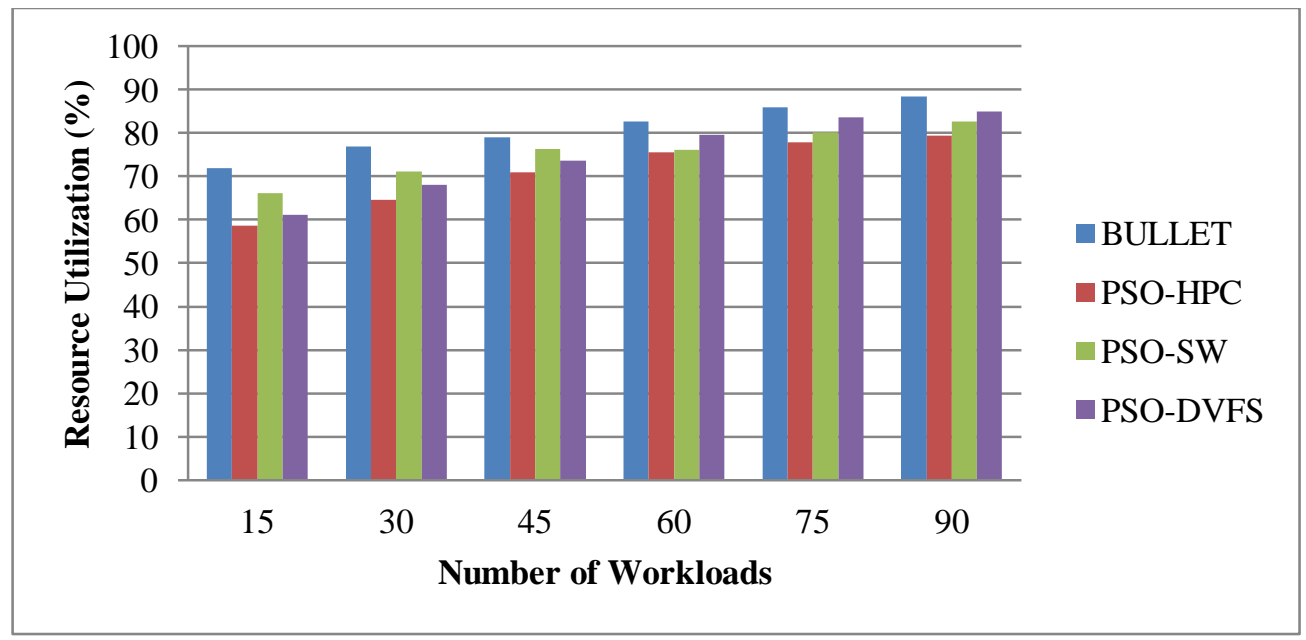

Figure 15: Effect of Change in Number of Workloads Submitted on Resource Utilization

\section{Test Case 10: Latency}

With increasing number of cloud workloads, the value of latency is increasing. The value of latency in BULLET is lesser as compared to PSO-HPC, PSO-SW and PSO-DVFS at different number of cloud workloads as shown in Figure 16. The minimum value of latency is 1.24 seconds at 15 cloud workloads and maximum is 9.13 seconds at 90 cloud workloads in BULLET. At 15 workloads, latency in BULLET is $2.23 \%$ lesser than PSO-HPC, $1.91 \%$ lesser than PSO-SW and 5.66\% lesser than PSO-DVFS but at 90 workloads, latency in BULLET is $6.11 \%$ lesser than PSO-HPC, $14.92 \%$ lesser than PSO-SW and $17.59 \%$ lesser than PSO-DVFS. 


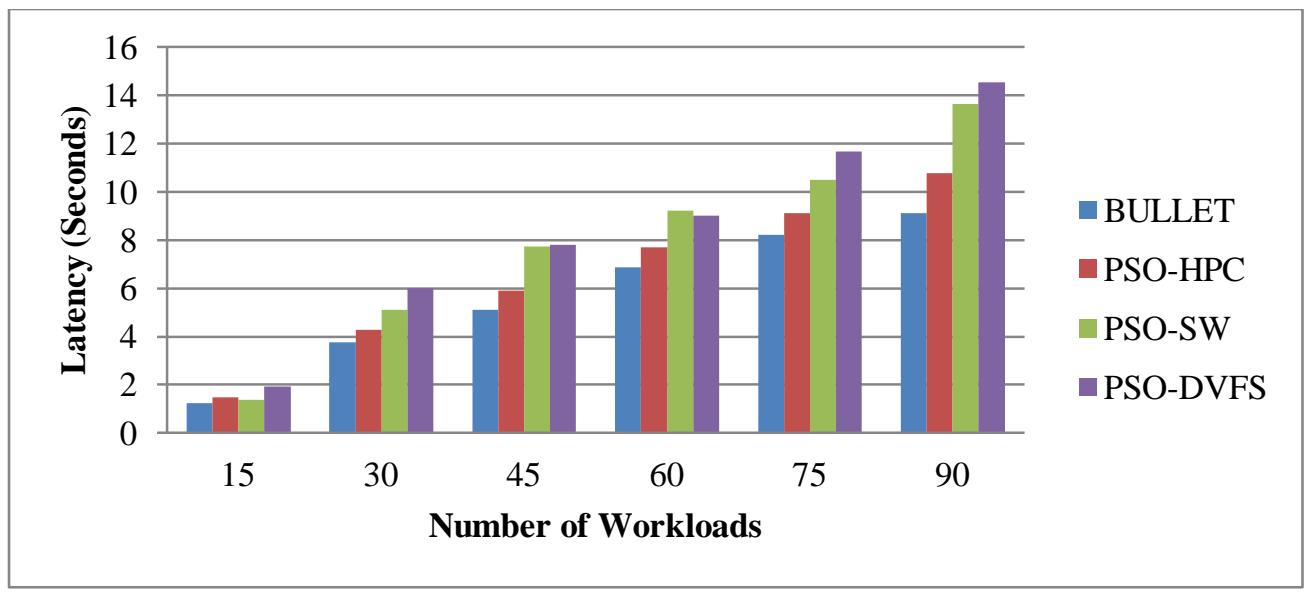

Figure 16: Effect of Change in Number of Workloads Submitted on Latency

\section{Test Case 11: Convergence of PSO}

Figure 17 plots the convergence of total cost computed by PSO over the number of iterations for different value of Resource Utilization (RU): $85 \%, 75 \%$ and $65 \%$ by executing different number of workloads. Initially the workloads are randomly initialized. Therefore, the total initial cost is very high at $0^{\text {th }}$ iteration. As the algorithm progresses, the convergence is drastic and achieves global minima very quickly. The number of iterations required for the convergence is seen to be 50-60, for our cloud environment.

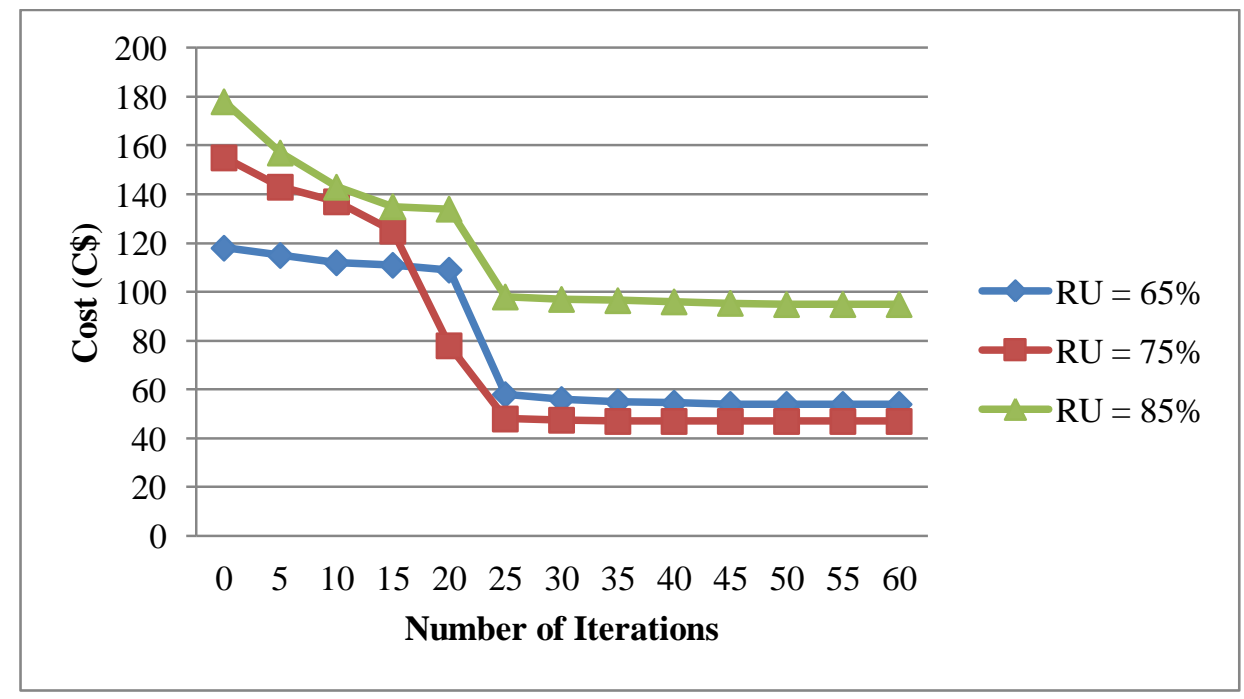

Figure 17: Convergence Curve of Total Cost

Table 5 describes the comparison of execution cost, execution time and energy consumption used to process same number of workloads (50 workloads of same type) on real cloud environment for PSO-HPC [14], PSO-SW [15] and PSO-DVFS [16]. In this experiment, we have considered three different cloud infrastructures with different processor configurations ( 2 core processor, 4 core processor, 8 core processor and 16 core processor) to measure the variation of execution cost, execution time and energy consumption. 
Table 5: Summary of Experimental Statistics on Real Cloud Environment

\begin{tabular}{|c|c|c|c|c|c|c|c|c|c|c|c|c|}
\hline \multirow{3}{*}{ Configuration } & \multicolumn{12}{|c|}{ QoS Parameter } \\
\hline & \multicolumn{4}{|c|}{ Execution Cost (C\$) } & \multicolumn{4}{|c|}{ Energy Consumption (kWh) } & \multicolumn{4}{|c|}{ Execution Time (Seconds) } \\
\hline & $\begin{array}{l}\text { PSO- } \\
\text { HPC }\end{array}$ & $\begin{array}{l}\text { PSO- } \\
\text { SW }\end{array}$ & $\begin{array}{l}\text { PSO- } \\
\text { DVFS }\end{array}$ & BULLET & $\begin{array}{l}\text { PSO- } \\
\text { HPC }\end{array}$ & $\begin{array}{l}\text { PSO- } \\
\text { SW }\end{array}$ & $\begin{array}{l}\text { PSO- } \\
\text { DVFS }\end{array}$ & BULLET & $\begin{array}{l}\text { PSO- } \\
\text { HPC }\end{array}$ & $\begin{array}{l}\text { PSO- } \\
\text { SW }\end{array}$ & $\begin{array}{l}\text { PSO- } \\
\text { DVFS }\end{array}$ & BULLET \\
\hline $\begin{array}{c}2 \text { Core } \\
\text { Processor }\end{array}$ & 102 & 78 & 49 & 37 & 145 & 118 & 77 & 55 & 321 & 356 & 301 & 229 \\
\hline $\begin{array}{c}4 \text { Core } \\
\text { Processor }\end{array}$ & 310 & 289 & 155 & 83 & 178 & 152 & 141 & 125 & 669 & 587 & 671 & 491 \\
\hline $\begin{array}{c}8 \text { Core } \\
\text { Processor }\end{array}$ & 451 & 371 & 299 & 151 & 415 & 372 & 237 & 195 & 1391 & 1423 & 1293 & 908 \\
\hline $\begin{array}{c}16 \text { Core } \\
\text { Processor }\end{array}$ & 522 & 439 & 391 & 203 & 564 & 456 & 389 & 225 & 1820 & 2109 & 2201 & 1423 \\
\hline
\end{tabular}

Figure 18, Figure 19, Figure 20, Figure 21, Figure 22, Figure 23 and Figure 24 describes the comparison of QoS parameters (execution cost, execution time, energy consumption, availability, resource utilization, latency and reliability) used to process different number of workloads (45 and 90) on cloud environment for BULLET with different number of Virtual Machines (VMs). The number of VMs used to execute the workloads was incremented gradually showing how the QoS parameters are optimized when more VMs were added to the cloud. As shown in Figure 18-24, with one virtual node running on Server R1, execution of 45 workloads finished in 636.12 seconds. With 12 virtual nodes (6 running on R1, 4 running on R2 and 2 running on R3), the application took 476.16 seconds. We note that the execution time is reduced by adding additional virtual nodes.

The value of reliability, availability, resource utilization, latency, execution time, execution cost and energy consumption has been calculated for 45 and 90 cloud workloads with different number virtual machines (VM nodes). By increasing the number of VMs, the percentage of reliability is increasing. The percentage of reliability with 45 workloads is more as compared to 90 Cloud workloads as shown in Figure 18. The maximum percentage of reliability is 94.65 at $12 \mathrm{VMs}$.

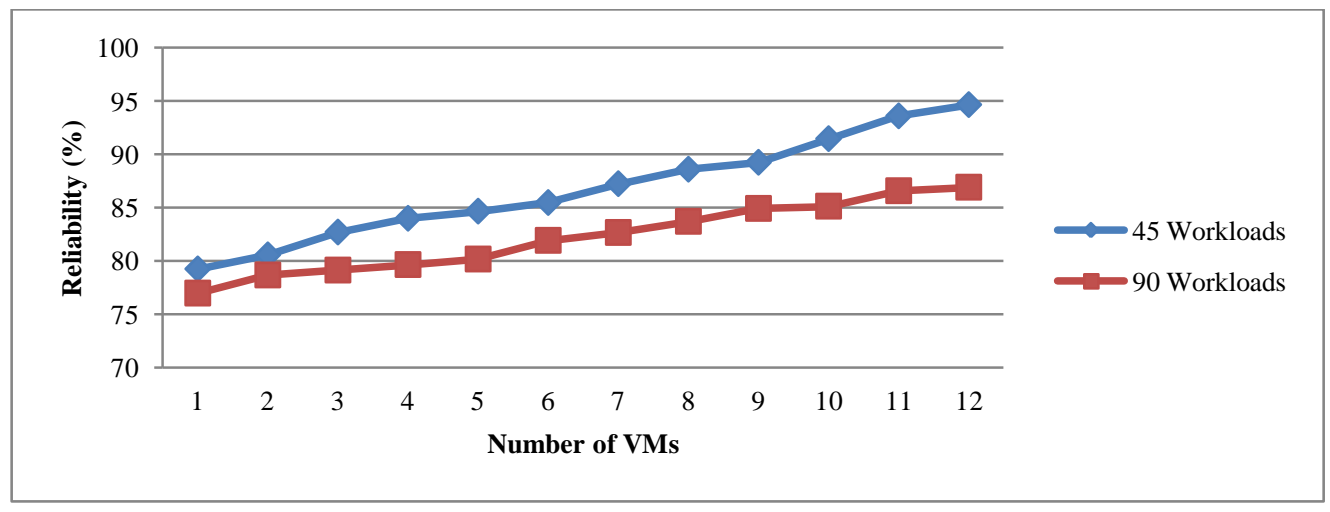

Figure 18: Effect of Change in Number of VMs on Reliability

By increasing the number of VMs, the percentage of availability is increasing as shown in Figure 19. The percentage of availability with 90 workloads is lesser as compared to 45 cloud workloads. The maximum percentage of reliability is 93.46 for 45 workloads and 92.44 for 90 workloads at 12 VMs. 


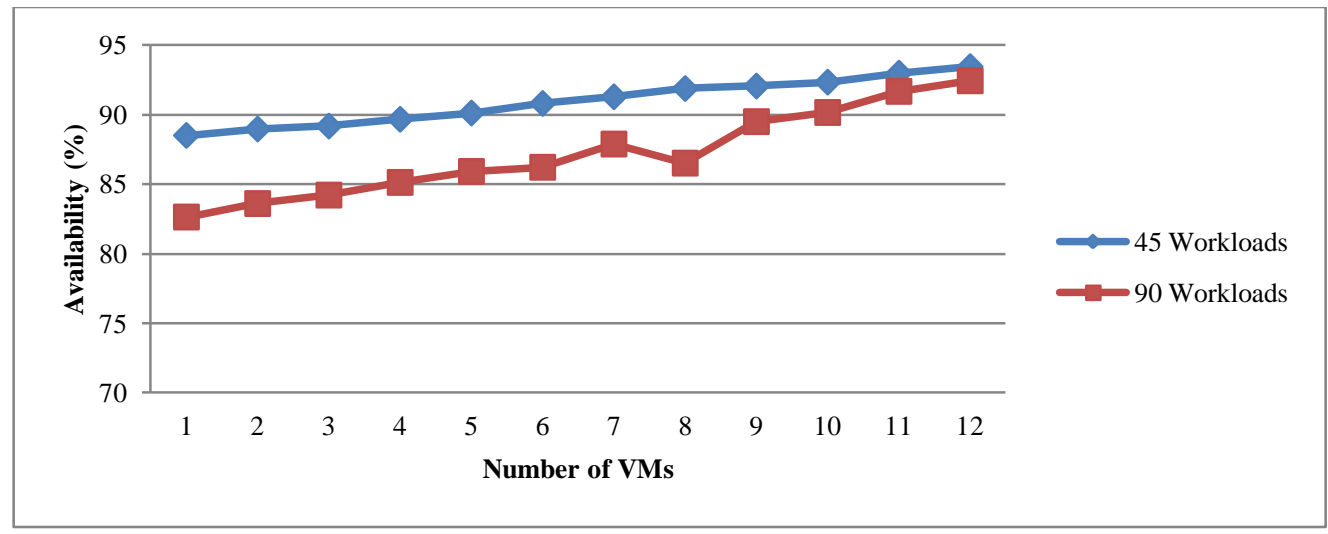

Figure 19: Effect of Change in Number of VMs on Availability

As shown in Figure 20, the percentage of resource utilization with 90 workloads is more as compared to 45 workloads. The maximum percentage of resource utilization is 89.68 at 90 workloads and minimum percentage is 88.41 at 45 workloads with $12 \mathrm{VMs}$.

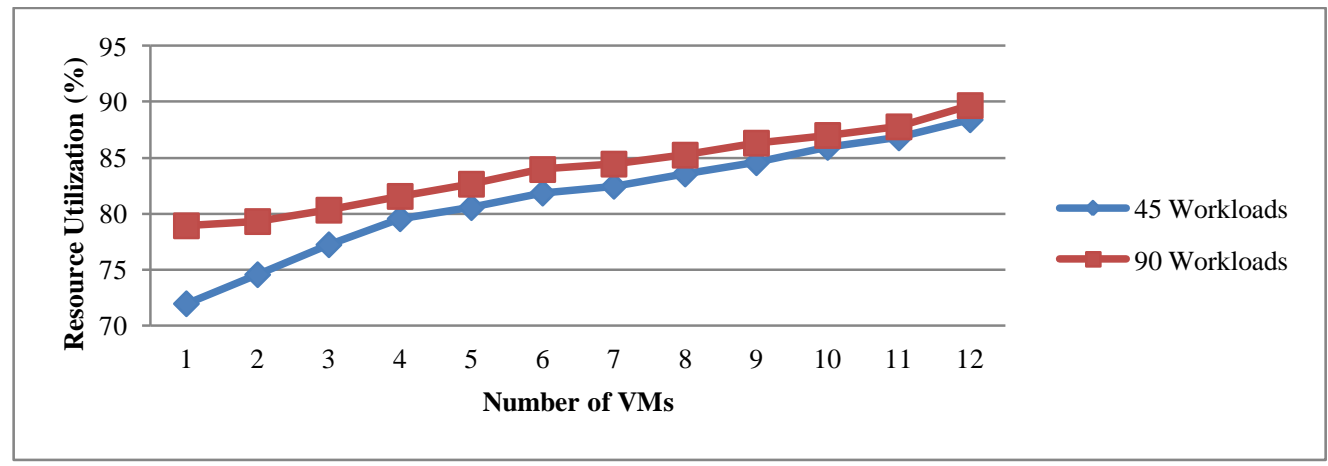

Figure 20: Effect of Change in Number of VMs on Resource Utilization

As shown in Figure 21, the execution time decreases with increase in number of VMs. At 45 workloads, execution time is lesser than 90 workloads. Figure 21 shows that the execution time reduces rapidly in 90 workloads as compared to 45 workloads.

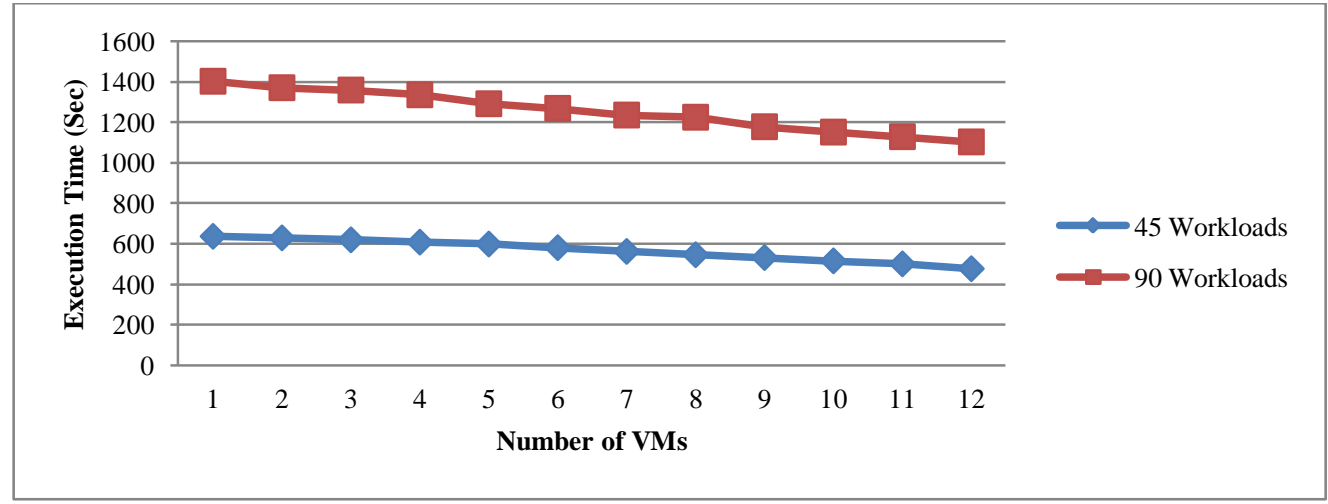

Figure 21: Effect of Change in Number of VMs on Execution Time 
With the increase in number of VMs, execution cost rises as shown in Figure 22. The minimum cost used is 47.58C $\$$ at 45 workloads and $322.31 \mathrm{C} \$$ at 90 workloads with $1 \mathrm{VM}$. The average value of execution cost at 45 workloads is $22.16 \%$ lesser than 90 workloads.

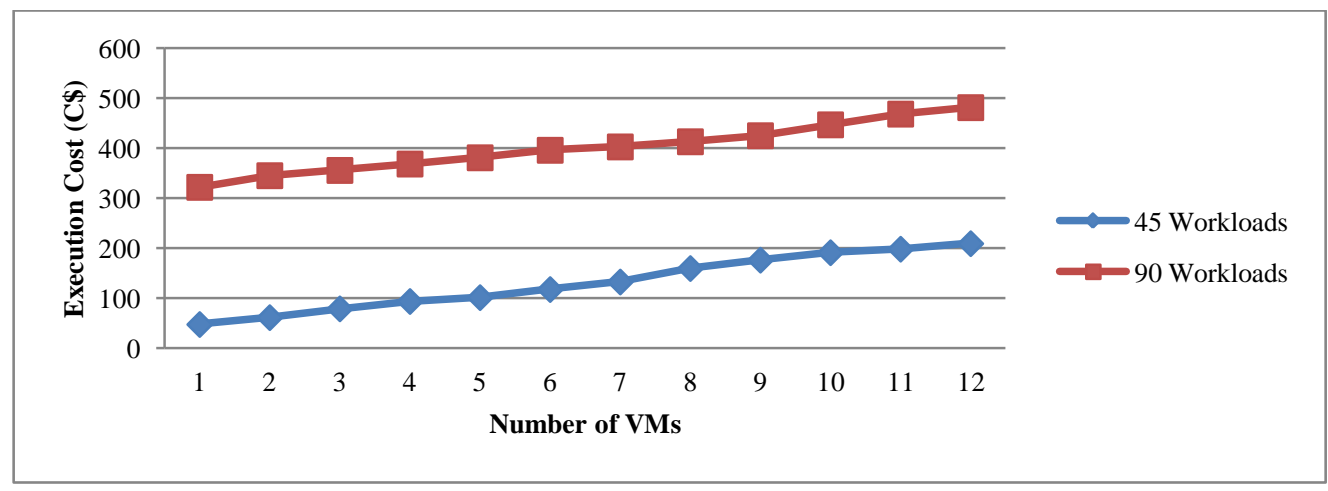

Figure 22: Effect of Change in Number of VMs on Execution Cost

With increasing the number of VMs, the value of latency is decreasing as shown in Figure 23. Initially, the value of latency is more for 90 workloads with $1 \mathrm{VM}$. At $12 \mathrm{VMs}$, maximum resources are utilized and value of latency for both 45 and 90 workloads is approximately same.

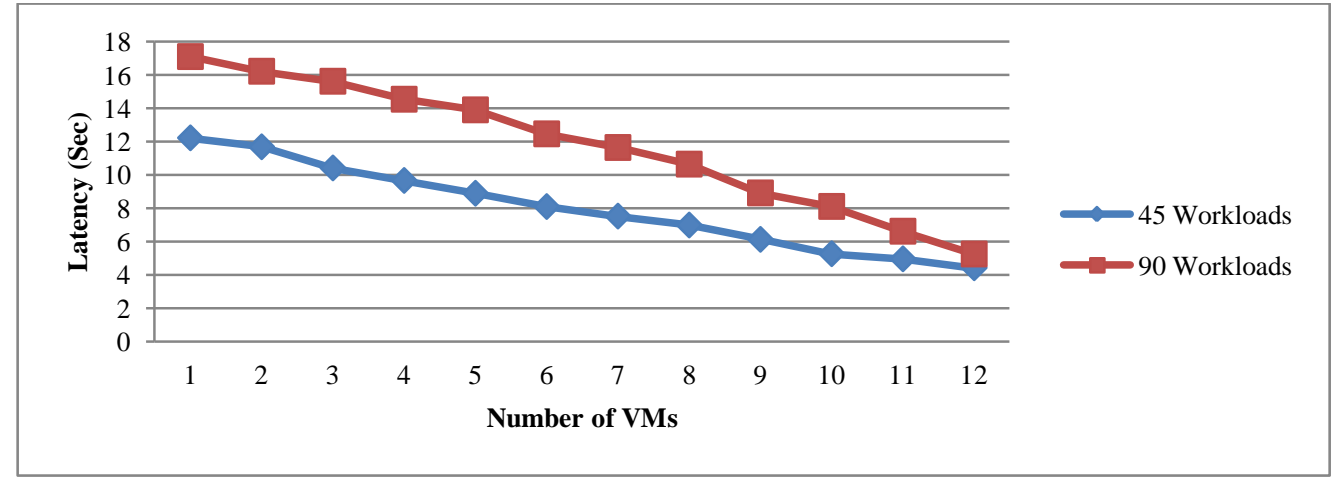

Figure 23: Effect of Change in Number of VMs on Latency

By increasing the number of VMs, the value of energy consumption increases as shown in Figure 24. The minimum value of energy consumption is $20.25 \mathrm{kWh}$ for 45 workloads at $1 \mathrm{VM}$. The average value of energy consumption with 45 workloads is $22.36 \%$ lesser 90 workloads.

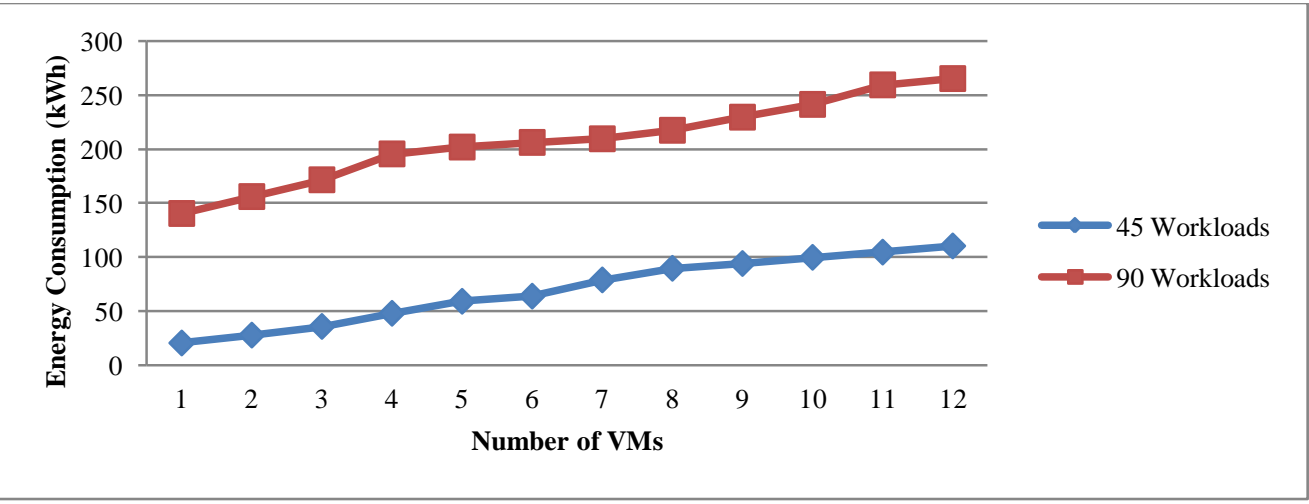

Figure 24: Effect of Change in Number of VMs on Energy Consumption 


\subsection{Statistical Analysis}

Statistical significance of the results has been analyzed by Coefficient of Variation $\left(c_{v}\right)$, a statistical method. $c_{v}$ is statistical measure of the distribution of data about the mean value. $c_{v}$ is used to compare to different means and furthermore offer an overall analysis of performance of the technique used for creating the statistics. It states the deviation of the data as a proportion of its average value, and is calculated as follows [Eq. 19]:

$$
c_{v}=\frac{S D}{\mathrm{M}} \times 100
$$

Where $S D$ is a Standard Deviation and $M$ is Mean. $c_{v}$ of execution time, cost and energy consumption has been studied of Cloud workload of proposed technique (BULLET) and existing algorithms (PSO-HPC, PSO-SW and PSO-DVFS) as shown in Figure 25, Figure 26 and Figure 27.

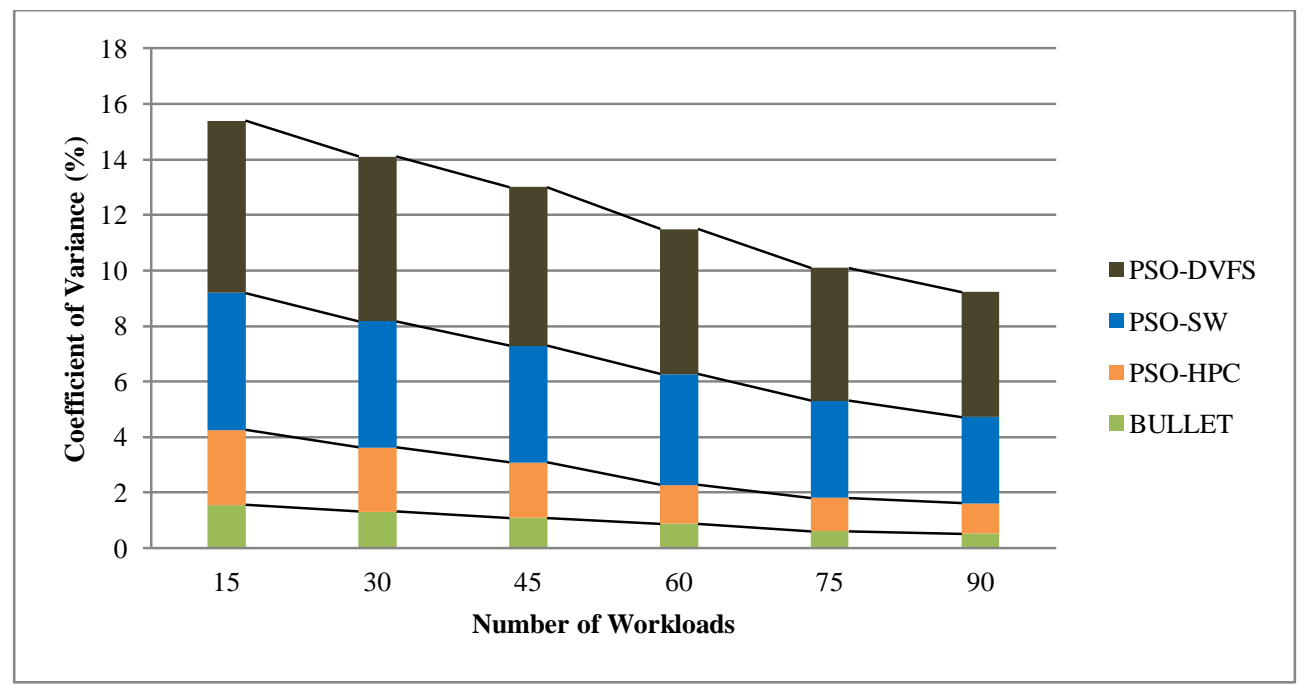

Figure 25: $c_{v}$ for Execution Time with each scheduling Algorithm

$c_{v}$ calculated for execution time and cost results attained by proposed algorithm and existing algorithms. Range of $c_{v}(0.25 \%-1.69 \%)$ for execution time, $(0.37 \%-1.96 \%)$ for cost and $(0.61 \%-2.47 \%)$ for energy consumption approves the stability of BULLET as shown in Figure 25, Figure 26 and Figure 27. Small value of $c_{v}$ signifies BULLET is more efficient in resource scheduling in the situations where the number of cloud workloads has changed. Value of $c_{v}$ decreases as the number of workloads is increasing. Statistical analysis demonstrates the

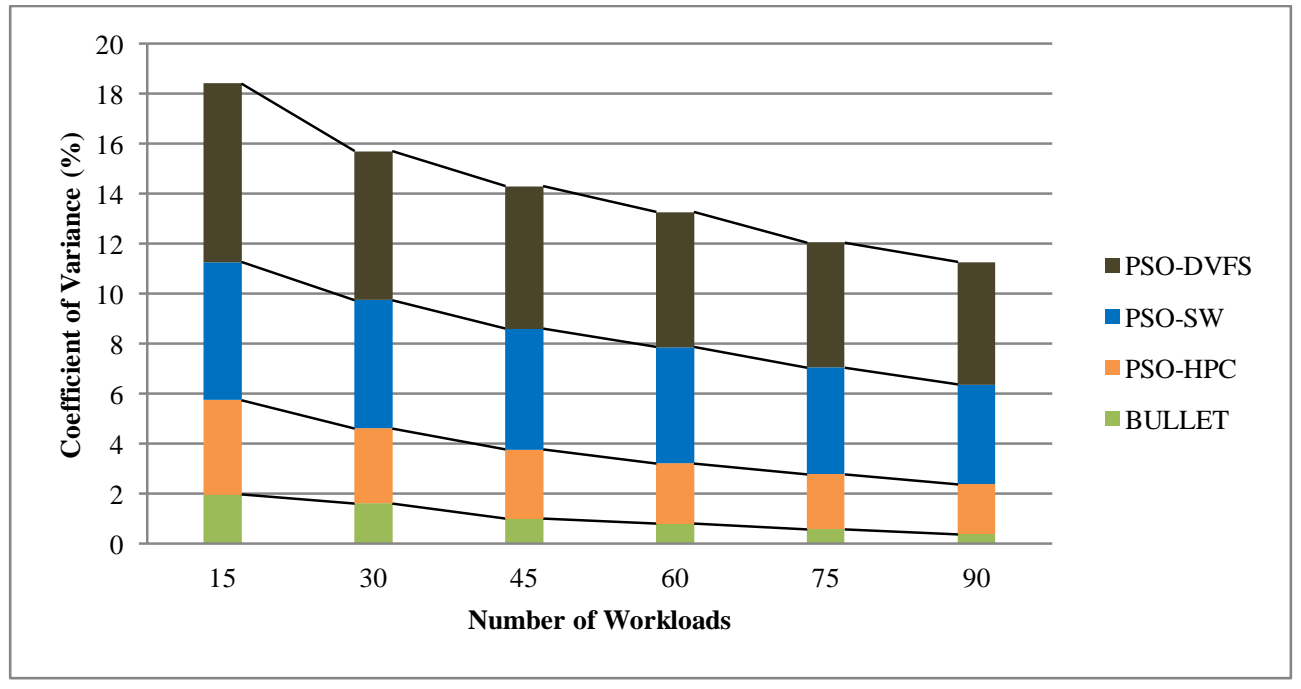

Figure 26: $c_{v}$ for Execution Cost with Each Scheduling Algorithm 


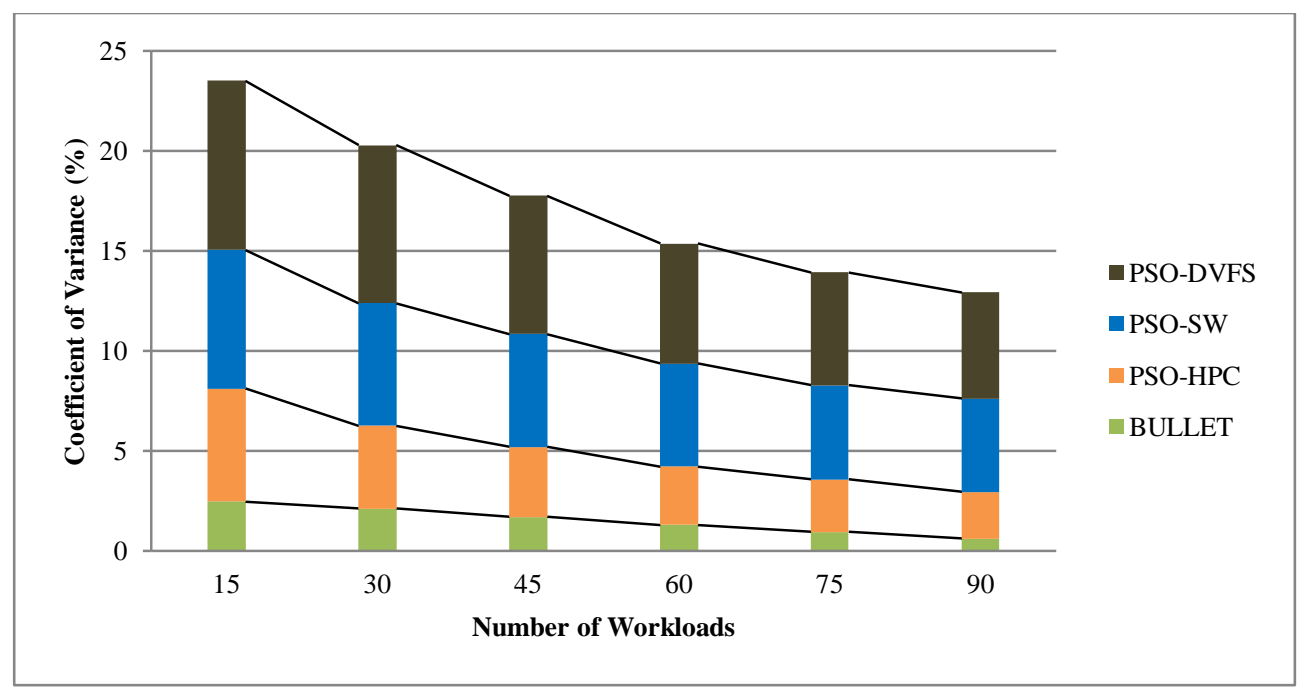

Figure 27: $c_{v}$ for Energy Consumption with Each Scheduling Algorithm

BULLET outperforms existing scheduling algorithms for large numbers of cloud workloads. With small value of $c_{v}$ system is more stable and BULLET attained the best results in the cloud for cost and execution time as QoS parameters.

The statistical analysis of QoS parameters (Figure 18-24) is described in Table 6. The number of samples considered is 12. The value of confidence interval is calculated using IBM SPSS 24. Table 6 lists the 95\% Confidence Intervals and the estimations of the medians of the differences of the values of QoS parameters of the servers with 45 and 90 workloads. From the Table 6, we can see that the estimated error (the pseudo-median of the differences) is less than $3 \%$ for all characteristics. This is representative of the fact, the mean value of all the QoS parameters with 95\% confidence interval from its lower value to upper value.

Table 6: Statistical Analysis of QoS Parameters

\begin{tabular}{|c|c|c|c|c|c|c|}
\hline \multirow[t]{2}{*}{ QoS Parameter } & \multirow[t]{2}{*}{$\begin{array}{l}\text { Number of } \\
\text { Workloads }\end{array}$} & \multirow[t]{2}{*}{$\begin{array}{l}\text { Standard } \\
\text { Deviation }\end{array}$} & \multirow{2}{*}{$\begin{array}{c}\text { Standard } \\
\text { Error } \\
\text { Mean }\end{array}$} & \multicolumn{2}{|c|}{$\begin{array}{l}\text { 95\% Confidence Interval } \\
\text { of the Difference }\end{array}$} & \multirow[t]{2}{*}{$\begin{array}{l}\text { Mean } \\
\text { Value }\end{array}$} \\
\hline & & & & Lower & Upper & \\
\hline \multirow[t]{2}{*}{ Execution Time (Sec) } & 45 & 53.34805 & 15.40026 & 532.6884 & 600.4799 & 566.5842 \\
\hline & 90 & 100.56821 & 29.03154 & 1190.6220 & 1318.4180 & 1254.52 \\
\hline \multirow[t]{2}{*}{ Execution Cost $(\mathrm{C} \$)$} & 45 & 55.75 & 16.09364 & 958.2748 & 166.1185 & 130.6967 \\
\hline & 90 & 49.34614 & 14.24500 & 369.6428 & 432.3489 & 400.9958 \\
\hline \multirow[t]{2}{*}{ Latency (Sec) } & 45 & 2.61545 & 0.75502 & 6.3633 & 9.6869 & 8.0251 \\
\hline & 90 & 3.90714 & 1.127889 & 9.2759 & 14.2408 & 11.7583 \\
\hline \multirow[t]{2}{*}{ Availability (\%) } & 45 & 1.65032 & 0.47641 & 89.8914 & 91.9886 & 90.94 \\
\hline & 90 & 3.19012 & 0.92091 & 85.1339 & 89.1877 & 87.1608 \\
\hline \multirow[t]{2}{*}{ Reliability (\%) } & 45 & 4.92129 & 1.42065 & 83.6493 & 89.9030 & 86.7762 \\
\hline & 90 & 3.29977 & 0.95256 & 80.6493 & 84.2875 & 82.1909 \\
\hline \multirow{2}{*}{$\begin{array}{c}\text { Resource Utilization } \\
(\%)\end{array}$} & 45 & 4.97449 & 1.43601 & 78.2951 & 84.6164 & 81.4557 \\
\hline & 90 & 3.45959 & 0.99870 & 81.7377 & 86.1340 & 83.9358 \\
\hline \multirow{2}{*}{$\begin{array}{c}\text { Energy Consumption } \\
(\mathrm{kWh})\end{array}$} & 45 & 31.37344 & 9.05673 & 49.2559 & 89.1234 & 69.1897 \\
\hline & 90 & 38.66417 & 11.16138 & 183.3145 & 232.4465 & 207.8805 \\
\hline
\end{tabular}

The variation of mean value of execution cost with different number of workloads and number of VMs is shown in Figure 28. With increasing the number of VMs, the execution cost increases. It is clearly shown that the execution cost for 15 workloads is $31.82 \%$ lesser than 90 workloads with 2 VMs. For 60 workloads, execution cost with 12 VMs is $22.61 \%$ more than $2 \mathrm{VMs}$. 


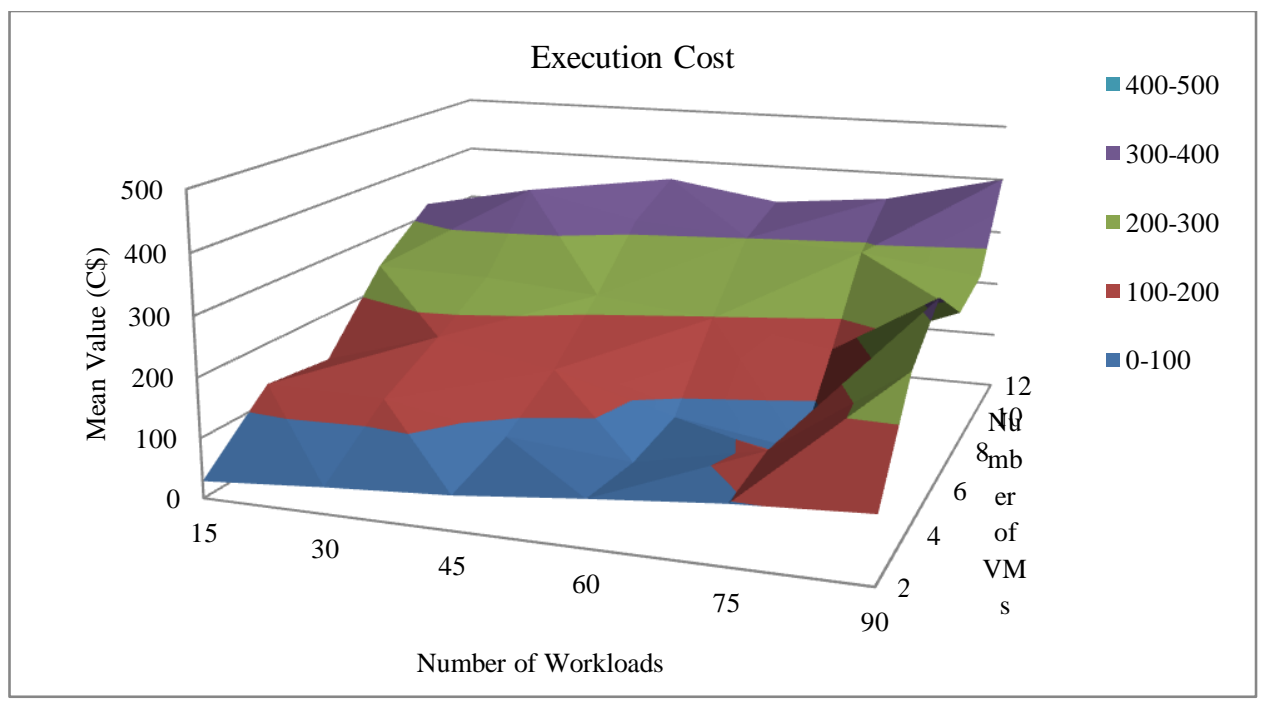

Figure 28: Statistical Analysis of Execution Cost

Figure 29 shows the variation of mean value of latency with different number of workloads and number of virtual nodes. It clearly shows the latency for 15 workloads is $42.52 \%$ lesser than 90 workloads with 2 VMs. With increasing the number of workloads, the latency increases but due to increasing in number of VMs, latency decreases. For 90 workloads, latency with 12 VMs is $27.92 \%$ lesser than 2 VMs.

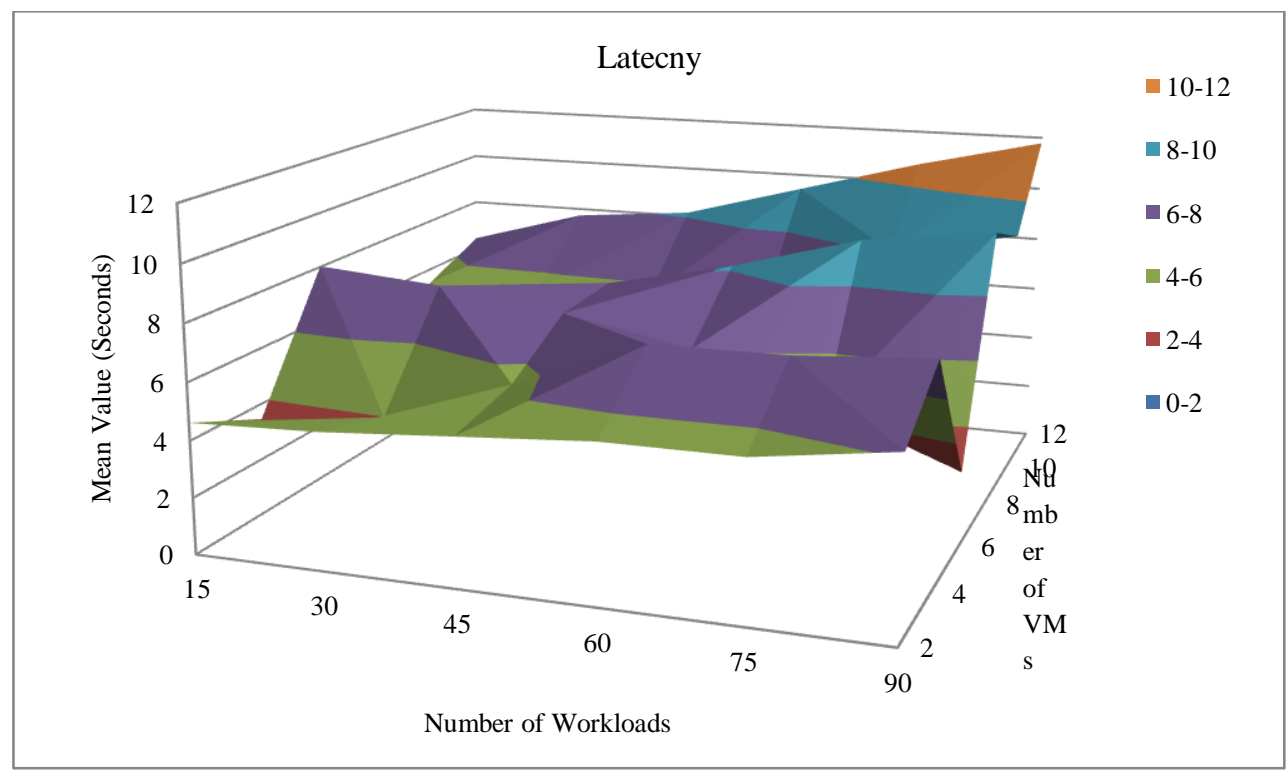

Figure 29: Statistical Analysis of Latency

The variation of mean value of availability with different number of workloads and number of VMs is shown in Figure 30. With increasing the number of VMs, the availability increases. It is clearly shown that the availability with $12 \mathrm{VMs}$ is $4.25 \%$ more than $2 \mathrm{VMs}$ for 15 workloads. With increasing the number of workloads, the availability decreases. The availability for 90 workloads is $7.52 \%$ lesser for 45 workloads with 12 VMs. 


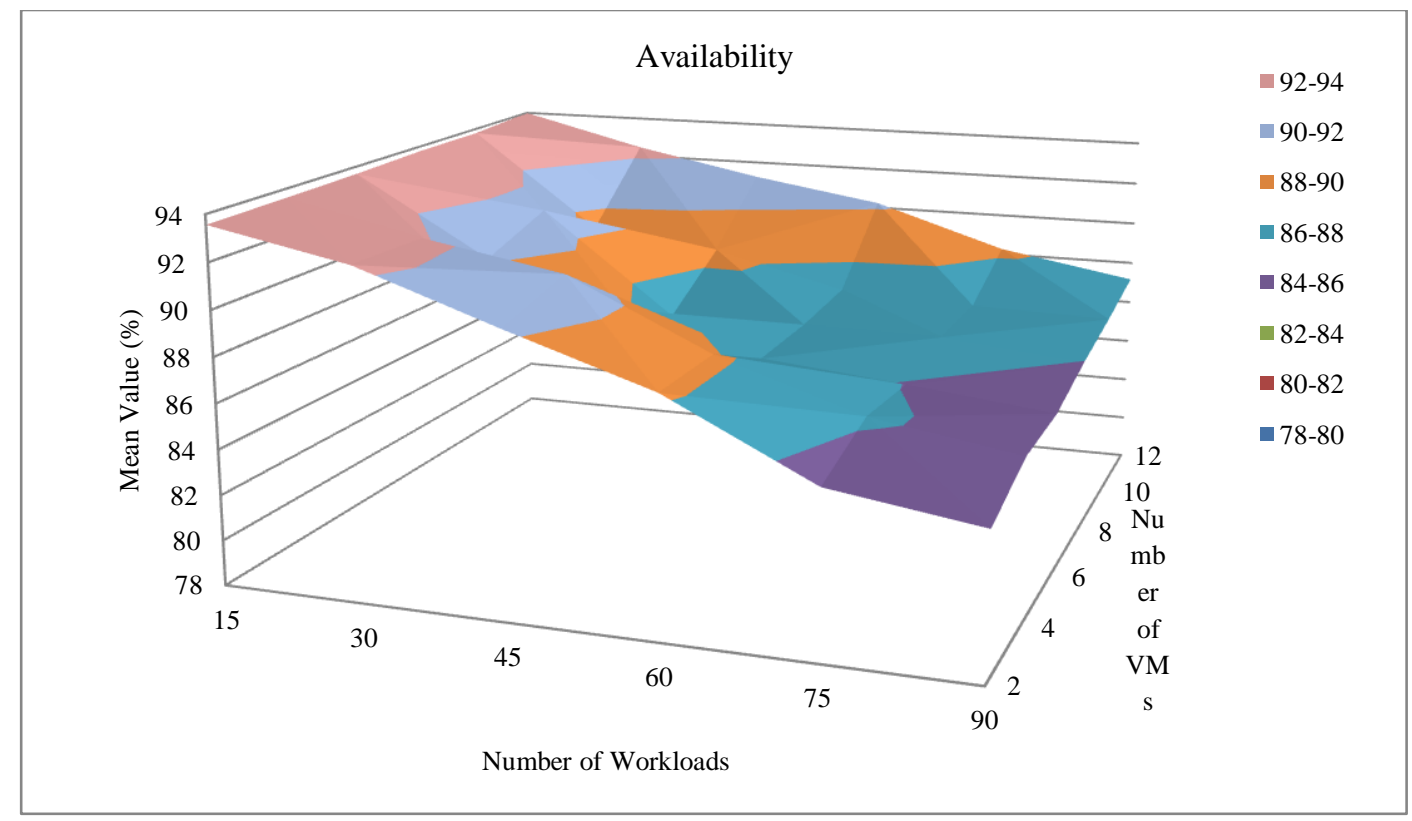

Figure 30: Statistical Analysis of Availability

Figure 31 shows the variation of mean value of reliability with different number of workloads and number of virtual nodes. With increasing the number of VMs, the reliability increases. It is clearly shown that the availability with 12 VMs is $7.61 \%$ more than $2 \mathrm{VMs}$ for 30 workloads. With increasing the number of workloads, the reliability decreases. The reliability for 75 workloads is $6.96 \%$ lesser for 15 workloads with 12 VMs.

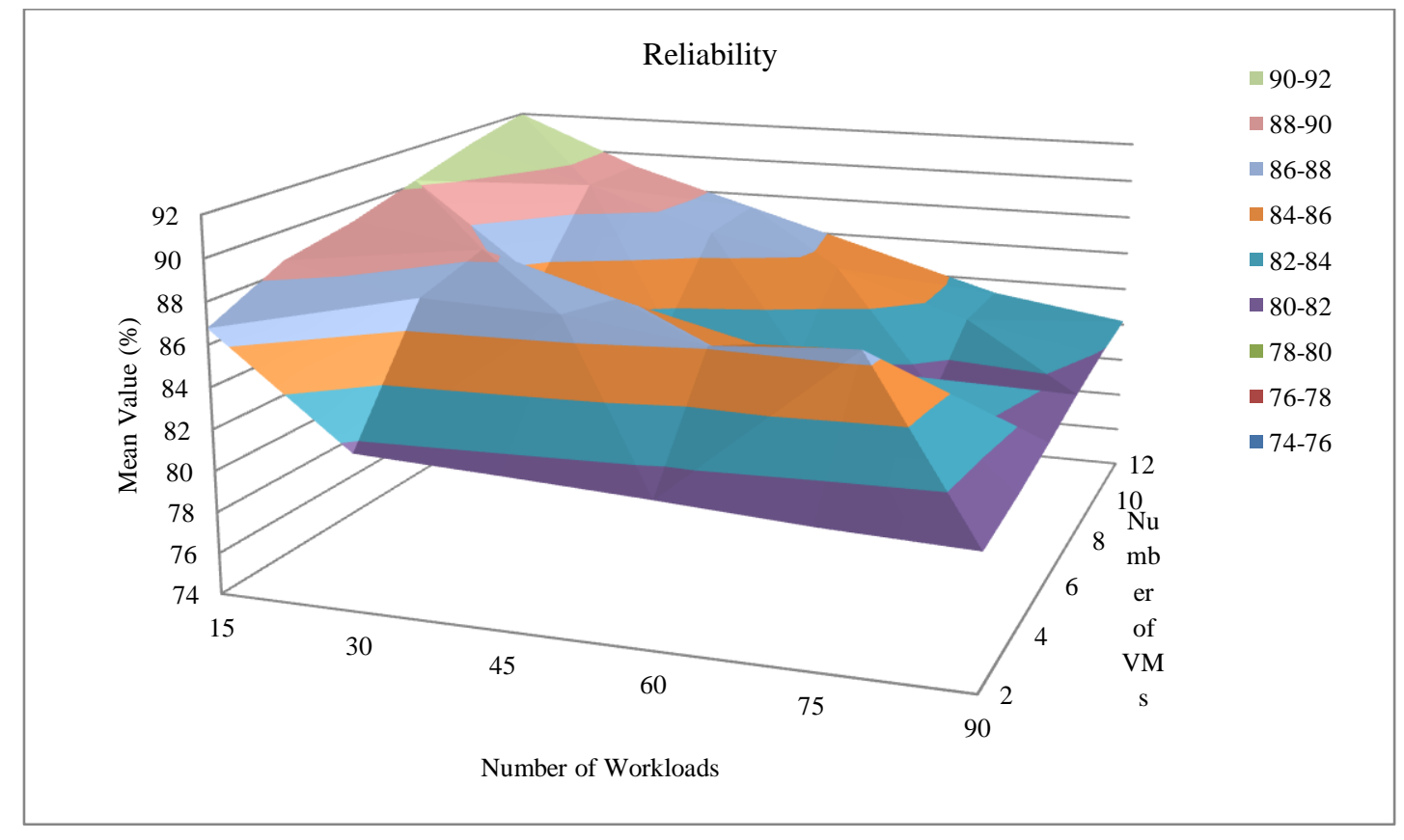

Figure 31: Statistical Analysis of Reliability 
The variation of mean value of resource utilization with different number of workloads and number of VMs is shown in Figure 32. With increasing the number of VMs, resource utilization decreases. It is clearly shown that the resource utilization for 30 workloads is $9.42 \%$ more than 75 workloads with 6 VMs. With increasing the number of workloads, resource utilization increases. For 60 workloads, resource utilization with 12 VMs is $4.61 \%$ lesser than 2 VMs.

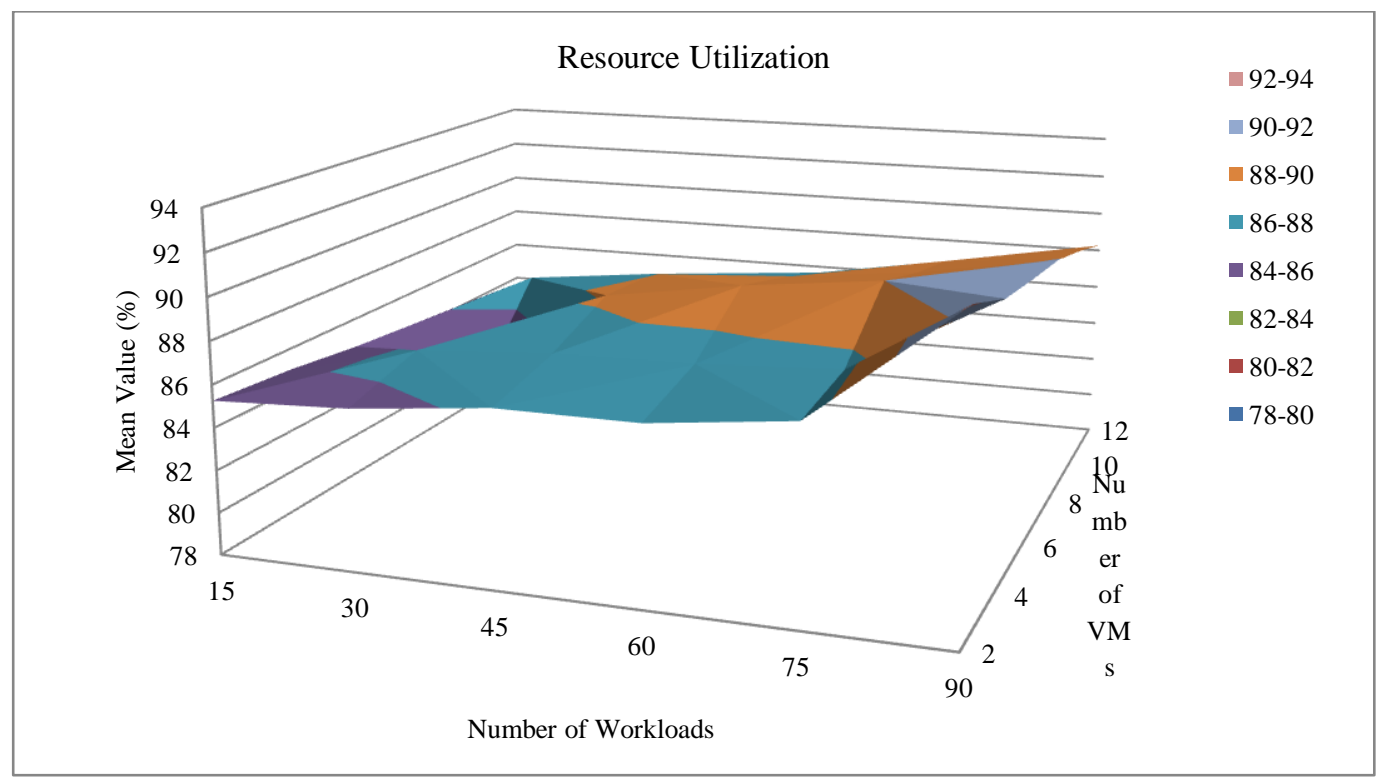

Figure 32: Statistical Analysis of Resource Utilization

Figure 33 shows the variation of mean value of energy consumption with different number of workloads and number of virtual nodes. It is clearly shown that the energy consumption increases with increasing the number of VMs and number of workloads. Energy consumption for 15 workloads is $14.75 \%$ lesser than 90 workloads with 2 VMs. With $12 \mathrm{VMs}$, energy consumption for 90 workloads is $17.56 \%$ more than 15 workloads.

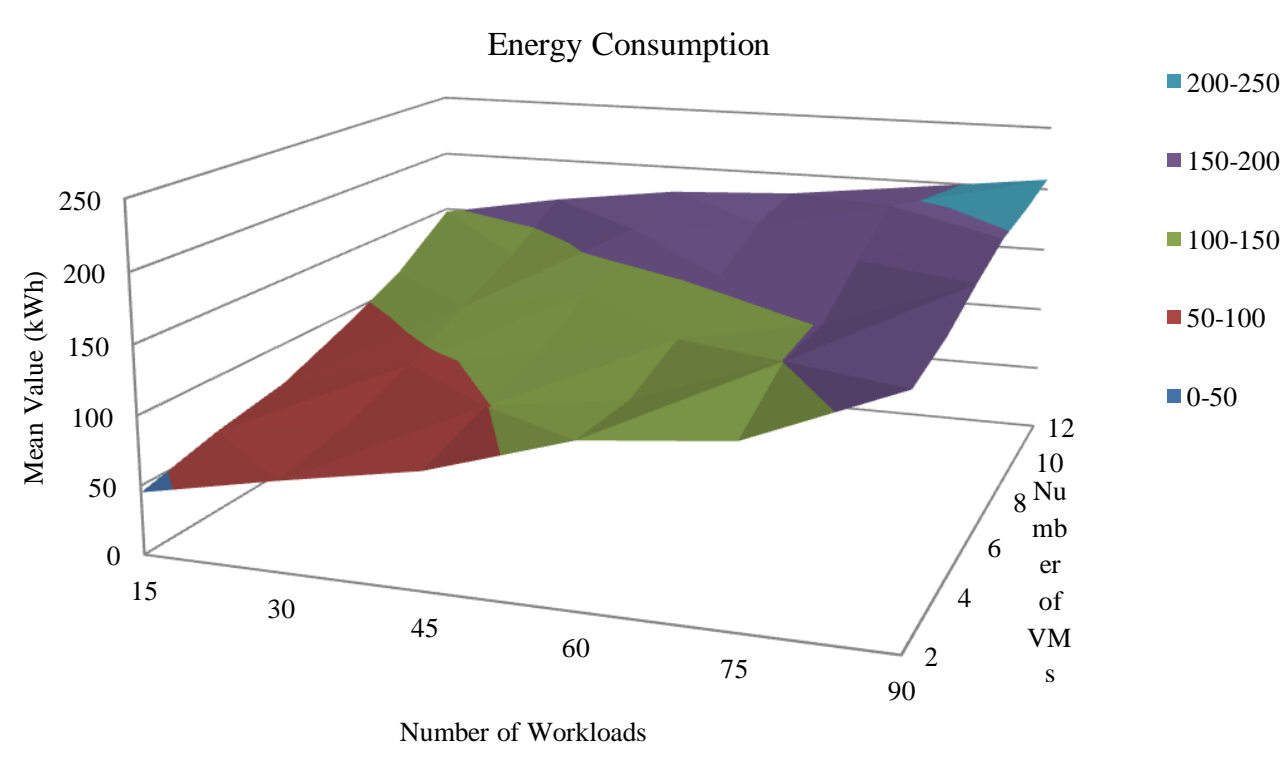

Figure 33: Statistical Analysis of Energy Consumption 
Figure 34 shows the variation of mean value of execution time with different number of workloads and number of virtual nodes (VMs). It clearly shows the execution time for 15 workloads is $34.152 \%$ lesser than 90 workloads with 2 VMs. With increasing the number of VMs, the execution cost decreases. For 90 workloads, execution time with 12 VMs is $25.44 \%$ lesser than 2 VMs.

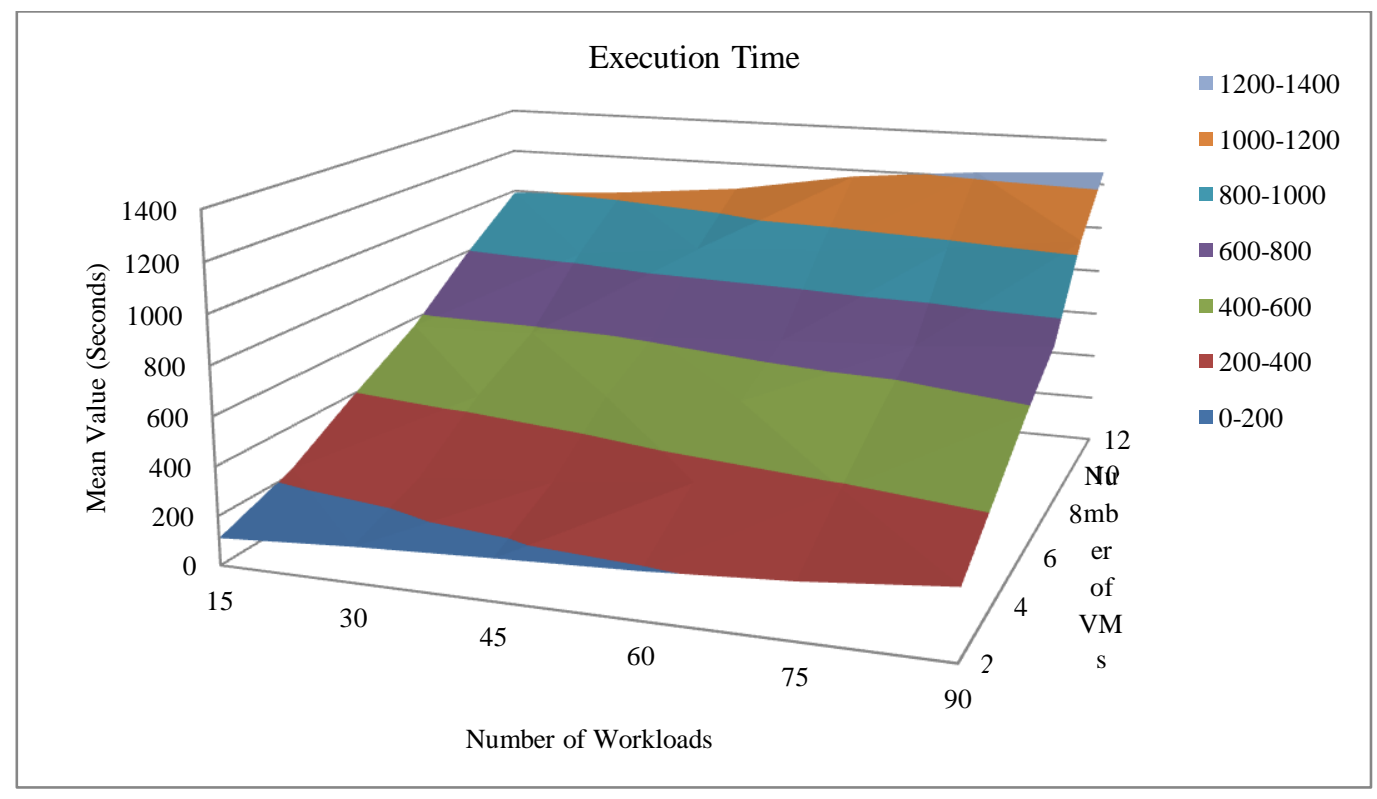

Figure 34: Statistical Analysis of Execution Time

\subsection{Discussions}

The performance of proposed PSO based resource scheduling technique (BULLET) has been compared with the existing scheduling algorithms (PSO-HPC, PSO-SW and PSO-DVFS). The performance of BULLET has been analyzed with different number of cloud workloads and resources. The performance of BULLET has been evaluated with respect to execution time, cost, energy and other QoS parameters like availability, reliability, latency and resource utilization. Execution cost permits the evaluation for selection of resources whereas duration of workload execution evaluates by execution time. The workload execution using the BULLET performs better as shown by all the experimental results. The overall cost for cloud consumer's workload execution is less. With the increase in budget, the more number of resources are provided to reduce the execution time. BULLET executes the same number of cloud workloads at maximum availability and reliability. The average value of energy consumption in BULLET is $7.61 \%, 11.45 \%$ and $17.19 \%$ lesser than PSO-HPC, PSO-SW and PSO-DVFS respectively. The minimum cost used in BULLET is $171 \mathrm{C} \$$ at 15 workloads and maximum is $416 \mathrm{C} \$$ at 90 workloads. The average value of execution cost in BULLET is 3.16\%, 4.72\% and 9.16\% lesser than PSO-HPC, PSO-SW and PSO-DVFS respectively.

At 30 workloads, execution time in BULLET is 6.69\% lesser than PSO-HPC, 7.12\% lesser than PSO-SW and 7.59\% lesser than PSO-DVFS. At 90 workloads, execution time in BULLET is $8.72 \%$ lesser than PSO-HPC, $11.39 \%$ lesser than PSO-SW and $14.79 \%$ lesser than PSO-DVFS. The maximum percentage of availability is $88.7 \%$ at minimum number of cloud workloads. At 75 workloads, percentage of availability in BULLET is $7.42 \%$ more than PSO-HPC, 9.91\% more than PSO-SW and $13.72 \%$ more than PSO-DVFS. The maximum percentage of reliability is 19.2 at 15 cloud workloads. At 60 workloads, percentage of reliability in BULLET is $4.91 \%$ more than PSO-HPC, $8.76 \%$ more than PSO-SW and $17.22 \%$ more than PSO-DVFS. The maximum percentage of resource utilization is $88.4 \%$ at 90 cloud workloads and minimum percentage is 71.96 at 15 workloads in BULLET. The minimum value of latency is 1.24 seconds at 15 cloud workloads and maximum is 9.13 at 90 cloud workloads in BULLET. At 15 workloads, latency in BULLET is 2.23\% lesser than PSO-HPC, 1.91\% lesser than PSO-SW and $5.66 \%$ lesser than PSO-DVFS but at 90 workloads, latency in BULLET is $6.11 \%$ lesser than PSO-HPC, $14.92 \%$ lesser than PSO-SW and $17.59 \%$ lesser than PSO-DVFS. Considering all these QoS parameters (execution time, 
cost, energy, availability, reliability, latency and resource utilization) and experimental result outcomes, it is shown that BULLET delivers a superior solution for heterogeneous cloud workloads and approximate optimum solution for challenges of resource scheduling.

\section{Conclusions and Future Scope}

In this paper, we have proposed PSO based resource scheduling technique called BULLET for scheduling of workloads in cloud environment so as to minimize the execution cost, time and energy. Experimental results demonstrate that BULLET is effective in decreasing the execution time, cost and energy consumption of cloud workloads along with other QoS parameters like availability, reliability, latency and resource utilization. Proposed scheduling technique provides effective outcomes as compared to existing PSO based scheduling algorithms at different levels of cost, time and energy as shown in test cases. Thus, resources can be scheduled easily and workloads can be executed effectively through proposed scheduling algorithm and this will further reduce queuing time which leads to effective resource execution. Proposed scheduling technique maps and executes the workloads based on workload details given by user and resource details given by providers. In future, we will further develop an autonomic resource management technique that efficiently schedules the provisioned cloud resources and maintains the SLA based on user's QoS requirements to reduce the above mentioned dependency. IaaS providers can use these results to quickly assess possible reductions in execution time and execution cost, hence having the potential to save energy. This framework can also be extended by identifying relationship between workload (patterns) and the resource demands (demands for compute, storage, and network resources) in the cloud.

\section{Acknowledgement}

One of the authors, Dr. Sukhpal Singh Gill [Post Doctorate Fellow], gratefully acknowledges the CLOUDS Lab, School of Computing and Information Systems, The University of Melbourne, Australia, for awarding him the Fellowship to carry out this research work.

\section{References}

[1] Moens H, Truyen E, Walraven S, Joosen W, Dhoedt B, De Turck F. Cost-effective feature placement of customizable multi-tenant applications in the cloud. Journal of Network and Systems Management. 2014 Oct 1;22(4):517-58.

[2] Sukhpal Singh and Inderveer Chana, "QoS-aware Autonomic Resource Management in Cloud Computing: A Systematic Review”, “ACM Computing Surveys", Volume 48, Issue 3, pp. 1-46, 2015.

[3] Sukhpal, Singh, and Inderveer Chana and Maninder Singh, "The Journey of QoS based Autonomic Cloud Computing", IT Professional Magazine, [IEEE] vol. 19, no. 2, pp. 42-49, 2017.

[4] Singh, Sukhpal, and Inderveer Chana. "Resource provisioning and scheduling in clouds: QoS perspective." The Journal of Supercomputing 72, no. 3 (2016): 926-960.

[5] Sukhpal Singh, and Inderveer Chana, "Q-aware: Quality of Service based Cloud Resource Provisioning", "Computers \& Electrical Engineering", [Elsevier], 47, pp. 138-160, 2015

[6] Sukhpal Singh, and Inderveer Chana, "QRSF: QoS-aware resource scheduling framework in cloud computing", "The Journal of Supercomputing”, [Springer], Vol. 71, no. 1, pp: 241-292, 2015.

[7] Varalakshmi, P., Aravindh Ramaswamy, Aswath Balasubramanian, and Palaniappan Vijaykumar. "An optimal workflow based scheduling and resource allocation in Cloud." In Advances in Computing and Communications, pp. 411-420. Springer Berlin Heidelberg, 2011.

[8] Xing, Li-Ning, Ying-Wu Chen, Peng Wang, Qing-Song Zhao, and Jian Xiong. "A knowledge-based ant colony optimization for flexible job shop scheduling problems." Applied Soft Computing 10, no. 3 (2010): 888-896.

[9] H. Topcuoglu, S. Hariri and M.-Y. Wu, "Task scheduling algorithms for heterogeneous processors," in Heterogeneous Computing Workshop, (HCW'99), San Juan, Puerto Rico, 1999.

[10] E.-S. T. El-kenawy, A. I. El-Desoky and M. F. Al-rahamawy, "Extended Max-Min Scheduling Using Petri Net and Load Balancing," International Journal of Soft Computing and Engineering (IJSCE), vol. 2, no. 4, pp. 198-203, 2012.

[11] K. Liu, H. Jin, J. Chen, X. Liu, . D. Yuan and Y. Yang, "A compromised-time-cost scheduling algorithm in swindew-c for instanceintensive cost-constrained workflows on a Cloud computing platform," International Journal of High Performance Computing Applications, vol. 24, no. 4, pp. 445-456, 2010

[12] A. Verma and S. Kaushal, "Deadline and Budget Distribution based Cost-Time Optimization Workflow Scheduling Algorithm for Cloud," in IJCA Proceedings on International Conference on Recent Advances and Future Trends in Information Technology (iRAFIT 2012), 2012.

[13] S. Pandey, L. Wu, S. Guru and R. Buyya, "A particle swarm optimization-based heuristic for scheduling workflow applications in Cloud computing environments," in Advanced Information Networking and Applications (AINA), 24th IEEE International Conference, Perth, Australia, 2010.

[14] Somasundaram, Thamarai Selvi, and Kannan Govindarajan. "CLOUDRB: A framework for scheduling and managing High-Performance Computing (HPC) applications in science cloud." Future Generation Computer Systems 34 (2014): 47-65. 
[15] Netjinda, Nuttapong, Booncharoen Sirinaovakul, and Tiranee Achalakul. "Cost optimal scheduling in IaaS for dependent workload with particle swarm optimization." The Journal of Supercomputing (2014): 1-25.

[16] Yassa, Sonia, Rachid Chelouah, Hubert Kadima, and Bertrand Granado. "Multi-objective approach for energy-aware workflow scheduling in cloud computing environments." The Scientific World Journal 2013 (2013).

[17] Chiang ML. Efficient diagnosis protocol to enhance the reliability of a cloud computing environment. Journal of Network and Systems Management. 2012 Dec 1;20(4):579-600.

[18] Rodrigo N. Calheiros, Christian Vecchiola, Dileban Karunamoorthy, and Rajkumar Buyya, "The Aneka platform and QoS-driven resource provisioning for elastic applications on hybrid Clouds", Future Generation Computer Systems, 28(6), (2012): 861 -870, 2012.

[19] Chen, Guo-chu, and Jin-shou Yu. "Particle swarm optimization algorithm." Information and Control-Shenyang- 34, no. 3 (2005): 318.

[20] Daniel LA, Madeira E, Medhi D. On Makespan, Migrations, and QoS Workloads' Execution Times in High Speed Data Centers. IEICE Transactions on Communications. 2015 Nov 1;98(11):2099-110.

[21] Lago D, Madeira E, Medhi D. High speed network impacts and power consumption estimation for cloud data centers. In Proceedings of the 30th Annual ACM Symposium on Applied Computing 2015 Apr 13 (pp. 615-620). ACM.

[22] Singh, Sukhpal, Inderveer Chana, Maninder Singh, and Rajkumar Buyya. "SOCCER: Self-Optimization of Energy-efficient Cloud Resources." Cluster Computing 19, no. 4 (2016): 1787-1800.

[23] S. Singh; I. Chana; R. Buyya, "STAR: SLA-aware Autonomic Management of Cloud Resources," in IEEE Transactions on Cloud Computing , pp.1-14, DOI: https://doi.org/10.1109/TCC.2017.2648788

\section{Author's Bibliography}

Sukhpal Singh Gill is faculty at Computer Science and Engineering Department, Thapar University, Patiala, India. Dr. Gill obtained Master Degree (Gold Medalist) and Doctoral Degree (DST Inspire Fellow) from Thapar University. Presently, Dr. Gill is working as a Post Doctorate Fellow at CLOUDS Lab, School of Computing and Information Systems, The University of Melbourne, Australia. His research interests include Software Engineering, Cloud Computing, Internet of Things and Fog Computing. He has more than 40 research publications in reputed journals and conferences.

Rajkumar Buyya is a Fellow of IEEE, Professor of Computer Science and Software Engineering and Director of the Cloud Computing and Distributed Systems (CLOUDS) Laboratory at the University of Melbourne, Australia. He is also serving as the founding CEO of Manjrasoft, a spin-off company of the University, commercialising its innovations in Cloud Computing. He has authored over 500 publications and four text books. He is one of the highly cited authors in computer science and software engineering worldwide (h-index 113+, 61000+ citations). He has served as the founding Editor-in-Chief (EiC) of IEEE Transactions on Cloud Computing and now serving as Co-EiC of Journal of Software: Practice and Experience.

Inderveer Chana joined Computer Science and Engineering Department of Thapar University, Patiala, India, in 1997 as Lecturer and is presently serving as Professor in the department. She is Ph.D. in Computer Science with specialization in Grid Computing. She has more than 100 research publications in reputed Journals and Conferences.

Maninder Singh received his Bachelor's Degree from Pune University in 1994, and holds a Master's Degree, with honors in Software Engineering from Thapar Institute of Engineering \& Technology, as well as a Doctoral Degree specialization in Network Security from Thapar University. Dr. Singh is currently working as Professor in Computer Science and Engineering Department at Thapar University.

Ajith Abraham received the Ph.D. degree in Computer Science from Monash University, Melbourne, Australia. He is currently the Director of Machine Intelligence Research Labs (MIR Labs), Scientific Network for Innovation and Research Excellence, USA. He is an author/co-author of $900+$ peer reviewed publications, h-index 68 and has over $25000+$ citations. 


\section{University Library}

\section{- M M N E R VA A gateway to Melbourne's research publications}

Minerva Access is the Institutional Repository of The University of Melbourne

Author/s:

Gill, SS;Buyya, R;Chana, I;Singh, M;Abraham, A

Title:

BULLET: Particle Swarm Optimization Based Scheduling Technique for Provisioned Cloud Resources

Date:

2018-04-01

Citation:

Gill, S. S., Buyya, R., Chana, I., Singh, M. \& Abraham, A. (2018). BULLET: Particle Swarm Optimization Based Scheduling Technique for Provisioned Cloud Resources. JOURNAL OF NETWORK AND SYSTEMS MANAGEMENT, 26 (2), pp.361-400. https://doi.org/10.1007/ s10922-017-9419-y.

Persistent Link:

http://hdl.handle.net/11343/283233 